\title{
Dynamical semigroups commuting with compact abelian actions
}

\author{
OLA BRATTELI AND DAVID E. EVANS \\ Institute of Mathematics, University of Trondheim, 7034 Trondheim - NTH, Norway ; \\ Mathematics Institute, University of Warwick, Coventry, CV4 7AL, England
}

(Received 1 May 1982 and revised 23 December 1982)

Abstract. Let $\mathscr{A}$ be a $C^{*}$-algebra and $\tau: G \rightarrow$ Aut $\mathscr{A}$ a compact abelian action such that the fixed point algebra $\mathscr{A}^{\tau}$ is simple. Denote by $\mathscr{A}_{F}$ the ${ }^{*}$-subalgebra of $G$-finite elements. Let $H: \mathscr{A}_{F} \rightarrow \mathscr{A}$ be a ${ }^{*}$-operator commuting with $\tau$ such that $\left.H\right|_{\mathscr{A}^{\tau}}=0$ and the matrix inequality

$$
\left[H\left(X_{i}^{*} X_{i}\right)\right] \leq\left[H\left(X_{i}\right)^{*} X_{j}+X_{i}^{*} H\left(X_{j}\right)\right]
$$

holds for all finite sequences $X_{1}, \ldots, X_{n}$ in $\mathscr{A}_{F}$. Then $H$ is closable, and the closure $\bar{H}$ is the generator of a strongly continuous semigroup $\{\exp (-t \bar{H}): t \geq 0\}$ of completely positive contractions. Furthermore, there exists a convolution semigroup $\left\{\mu_{t}: t \geq 0\right\}$ of probability measures on $G$ such that

$$
\exp (-t \vec{H})(X)=\int_{G} d \mu_{\mathrm{t}}(g) \tau(g)(X), \quad t \geq 0, \quad X \in \mathscr{A} .
$$

This result has various extensions and refinements.

\section{Introduction}

In [1, appendix C] a theorem called Robert's version of Tannaka duality is proved. A special case of this, which could be called Roberts's version of Pontryagin duality, reads as follows:

Let $G$ be a compact abelian group and $\tau$ an automorphic action of $G$ on a von Neumann algebra $\mathcal{M}$, such that the fixed point algebra $\mathcal{M}^{\tau}$ is a factor. Let $\alpha$ be an automorphism of $\mathscr{M}$ such that

$$
\begin{array}{ll}
\text { (1.1) } \alpha \tau(g)=\tau(g) \alpha & \text { for all } g \text { in } G ; \\
\text { (1.2) } \alpha(X)=X & \text { for all } X \text { in } \mathcal{M}^{\tau} .
\end{array}
$$

Then there exists a $g \in G$ such that $\alpha=\tau(g)$.

In this paper we replace the automorphism $\alpha$ with a general completely positive map $S$ satisfying (1.1) and (1.2). It turns out that the extremal such maps are just the automorphisms $\tau(G)$, and in general $S$ has the decomposition

$$
S=\int_{G} d \mu(g) \tau(g)
$$

where $\mu$ is a probability measure on $G$. This result remains true if $\mu$ is replaced 
by a $C^{*}$-algebra $\mathscr{A}$ such that $\mathscr{A}^{\tau}$ is simple. With somewhat less restrictive assumptions on $\tau$, the measure $\mu$ is replaced by a measure taking values in the centre of the multiplier algebra of $\mathscr{A}^{\tau}$. These results are contained in $\S 4$.

In $\$ 5$ we study generators of dynamical semigroups of completely positive maps. This paper was in part inspired by [9] where it was proved that if $G$ is a compact abelian group acting ergodically on a simple $C^{*}$-algebra $\mathscr{A}$, and $\delta$ is a derivation defined on the $C^{\infty}$-vectors, then $\delta$ has a unique decomposition

$$
\delta=\delta_{0}+\tilde{\delta}
$$

where $\delta_{0}$ is the generator of a one-parameter subgroup of $G$, and $\tilde{\delta}$ is approximately inner. The derivation $\delta_{0}$ is nothing but the invariant part of $\delta$,

$$
\delta_{0}=\int d g \tau(g) \delta \tau(-g)
$$

That $\delta_{0}$ generates a one-parameter subgroup of $\tau$ is then closely related to Roberts's version of Pontryagin duality. In order to establish analogous decompositions for generators of completely positive semigroups, we first need to characterize those generators commuting with the ergodic actions, and this is done in corollary 5.8. It is a remarkable consequence of complete positivity that such a generator has the same form whether the $C^{*}$-algebra $\mathscr{A}$ is abelian or not. If $G=\mathbb{V}^{d}$, it is a sum of three terms: the first is a linear combination of the elements in a basis

$$
\frac{\partial}{\partial t_{i}}, \quad i=1,2, \ldots, d,
$$

for the action of the Lie algebra of $G$ on $\mathscr{A}$; the second, the negative of an elliptic operator in $\partial / \partial t_{i}, i=1,2, \ldots$; and the third a bounded superposition of operators of the form

$$
-\exp \left(\sum_{i=1}^{d} x_{i} \frac{\partial}{\partial t_{i}}\right),
$$

given by the Lévy-Khinchin formula.

Ideally, one would like to characterize generators of actions

$$
\left\{S_{t}=\exp (-t H): t \geq 0\right\}
$$

on a $C^{*}$-algebra $\mathscr{A}$ with the properties

(1.3) $S_{t}$ is (completely) positive for all $t \geq 0$;

(1.4) $S_{t} \tau(g)=\tau(g) S_{i}, \quad$ for all $t \geq 0, g$ in $G$.

(1.5) $S_{t}(X)=X, \quad$ for all $X$ in the fixed point algebra $\mathscr{A}^{\tau}$.

It has recently been proved that a closed derivation $\delta$ satisfying

$$
\delta \tau(g)=\tau(g) \delta \text {, and }\left.\delta\right|_{A^{r}}=0,
$$

is automatically the generator of a one parameter group of *-automorphisms [6]; see [17], [18], [20], [22], [27], [28] for related results. However there does not seem to be a simple algebraic condition replacing the derivation property which characterizes the generators of completely positive semigroups, since the domain 
$D(H)$ of such an operator is not necessarily an algebra [8]. One can replace algebraic properties with analytic ones, and the following result is true for rather trivial reasons.

Let $\tau$ be an action of a compact (abelian) group $G$ on a $C^{*}$-algebra $\mathscr{A}$. Suppose $H$ is an operator on $\mathscr{A}$ such that $D(H)=\mathscr{A}_{F}$, the $G$-finite elements of $\mathscr{A}$, satisfying:

(1.6) $H$ is dissipative, i.e. if $X \in \mathscr{A}_{F}$, there exists a non-zero $\eta \in \mathscr{A}^{*}$, such that

$$
\eta(X)=\|\eta\|\|X\|
$$

and

(1.7) $H \tau(g)=\tau(g) H \quad$ for all $g$ in $G$;

(1.8) $H(X)=0$ for all $X$ in $\mathscr{A}^{\top}$.

Then $H$ is closable, and the closure generates a semi-group of positive maps.

The proof follows in part an argument from [17]. As $H$ is dissipative it is closable, and its closure $\bar{H}$ is dissipative [7, lemma 3.1.14]. Condition (1.7) ensures that $H$ maps the spectral subspace

$$
\mathscr{A}^{\tau}(\gamma)=\{X \in \mathscr{A}: \tau(g) X=\langle\gamma, g) X, \text { for all } g \in G\}
$$

into itself, for any $\gamma \in \hat{G}$. The closed graph theorem shows that the restriction of $H$ to $\mathscr{A}^{\tau}(\gamma)$ is bounded, and so the elements of $\mathscr{A}^{\tau}(\gamma)$ are analytic vectors for $\bar{H}$. By [7, lemma 3.1.15, theorem 3.1.19], $\bar{H}$ generates a semigroup of contractions

$$
\left\{S_{t}=\exp (-t \bar{H}), t \geq 0\right\} .
$$

The semigroup $S$ acts trivially on $\mathscr{A}^{\tau}$ and by the first lemma of $\S 4$, any approximate identity for $\mathscr{A}^{\tau}$ is also an approximate identity for $\mathscr{A}$. Since this approximate identity converges to 1 in the universal enveloping algebra $\mathscr{A}^{* *}$, it follows that

$$
S_{t}^{* *} 1=1, \quad \text { for each } t \geq 0 .
$$

But each $S_{t}^{* *}$ is a contraction, and so is positive [7, corollary 3.2.6], and hence each $S_{t}$ is positive.

In $\S 5$ we prove versions of this result where $(1.6)$ is replaced by the algebraic condition:

$$
\left[H\left(X_{i}^{*} X_{j}\right)\right] \leq\left[H\left(X_{i}\right)^{*} X_{j}+X_{i}^{*} H\left(X_{j}\right)\right]
$$

for all finite sequences $X_{1}, X_{2}, \ldots$, in the linear span of the spectral subspaces. We have to impose some restrictions on the action $\tau$ in order to make our proofs work, but these situations include the ergodic actions. Other results have been obtained in [21] when the algebra is abelian.

If $\mathscr{A}$ is a $C^{*}$-algebra on a Hilbert space $\mathscr{H}$, it is known [11] that the bounded generator $H$ of a norm-continuous semigroup of completely positive maps can be expressed as

$$
H(X)=-K(X)+L X+X L^{*},
$$

where $L \in \mathscr{A}^{\prime \prime}$, and $K$ is completely positive. In the last remark in $\$ 5$, we establish an analogous decomposition for the semigroups we consider, but $K(X)$ may be 
unbounded for all $X \neq 0$ in $D(H)$. Finally $\S 6$ contains some examples; in particular the ergodic action of $\mathbb{Z}_{2} \times \mathbb{Z}_{2}$ on $M_{2}$ is analysed in detail.

\section{Preliminaries}

If $G$ is a compact abelian group, an action $\tau$ of $G$ on a $C^{*}$-algebra $\mathscr{A}$ will be a homomorphism $\tau$ from $G$ into $\operatorname{Aut}(\mathscr{A})$, the group of all ${ }^{*}$-automorphisms of $\mathscr{A}$, which is strongly continuous in the sense that

$$
g \rightarrow \tau(g)(X)
$$

is norm continuous for each $X$ in $\mathscr{A}$. If $\gamma \in \hat{G}$, the dual group of $G, P_{\gamma}$ denotes the norm one projection

$$
\int d g(\overline{\gamma, g}) \tau(g)
$$

on $\mathscr{A}$ so that

$$
P_{\gamma} P_{\gamma^{\prime}}=0 \quad \text { if } \gamma \neq \gamma^{\prime}
$$

and

$$
\operatorname{Sp}(\tau)=\left\{\gamma \in \hat{G}: P_{\gamma} \neq 0\right\} .
$$

The ranges are denoted by

$$
\mathscr{A}^{\tau}(\gamma)=P_{\gamma} \mathscr{A},
$$

the spectral subspace corresponding to $\gamma$, and we often write $P$ for $P_{0}$ and

$$
\mathscr{A}^{\top}=\mathscr{A}^{\tau}(0)
$$

for the fixed point algebra. Then

$$
\mathscr{A}^{\tau}(\gamma)=\{X \in \mathscr{A}: \tau(g)(X)=\langle\gamma, g\rangle X, \quad \forall g \in G\},
$$

and

$$
\mathscr{A}^{\tau}\left(\gamma_{1}\right) \mathscr{A}^{\tau}\left(\gamma_{2}\right) \subseteq \mathscr{A}^{\tau}\left(\gamma_{1}+\gamma_{2}\right), \quad \mathscr{A}^{\tau}(\gamma)^{*}=\mathscr{A}^{\tau}(-\gamma) .
$$

An element $X \in \mathscr{A}$ lies in the linear span $\left\{\mathscr{A}^{\tau}(\gamma): \gamma \in \hat{G}\right\}$ if and only if the linear $\operatorname{span}\{\tau(g)(X): g \in G\}$ is finite dimensional. Such elements are called $G$-finite, and the $G$-finite elements

form a dense ${ }^{*}$-subalgebra of $\mathscr{A}$.

$$
\mathscr{A}_{F}^{\tau}=\mathscr{A}_{F}
$$

For a von Neumann algebra $\mathscr{A}=\mathscr{M}$, the functions $g \rightarrow \tau(g)(X)$ are only required to be $\sigma$-weakly continuous. The set of $X \in \mathcal{M}$ such that $g \rightarrow \tau(g)(X)$ is strongly continuous form a $\sigma$-weakly dense norm-closed ${ }^{*}$-subalgebra $\mathscr{M}_{0}$ of $\mathscr{M}$ which is called the strong continuity subspace of $\tau . \mathscr{M}_{0}$ is the norm closure of $\mathcal{M}_{F}$, [7], [26].

If $\mathscr{A}$ is a $C^{*}$-algebra, $M(\mathscr{A})$ will denote its multiplier algebra, and $K(\mathscr{A})$ the Pedersen or minimal dense ideal $[26,3.12,5.6]$.

If $\mathscr{A}$ is a $C^{*}$-algebra, $\mathscr{K}$ a Hilbert space and

$$
Q=\mathscr{A} \rightarrow B(\mathscr{K})
$$

a completely positive linear map, then the Stinespring decomposition of $Q$ consists of a triple $(\pi, \mathscr{H}, V)$ where $\pi$ is a representation of $\mathscr{A}$ on a Hilbert space $\mathscr{H}$, 
$V: \mathscr{H} \rightarrow \mathscr{K}$ is a bounded linear operator such that

$$
Q(X)=V^{*} \pi(X) V \quad X \in \mathscr{A},
$$

and $V \mathscr{K}$ is cyclic for $\pi,[3],[16]$.

\section{Centre-valued measures}

In order to formulate our main theorems, we need some results on vector valued measures, which are straightforward, but do not seem easily available in the literature. We must make sense of expressions like

$$
\int_{G} d \mu(g) X(g)
$$

when $G$ is a compact Hausdorff space, $X$ is a continuous function from $G$ into a $C^{*}$-algebra $\mathscr{A}$, and $\mu$ is a measure on $G$ taking values in an abelian $C^{*}$-algebra $\mathscr{C}$ which is a subalgebra of the centre of the multiplier algebra $M(\mathscr{A})$ of $\mathscr{A}$, such that $\mathscr{C}$ contains the identity of $M(\mathscr{A})$.

We define a $\mathscr{C}$-valued probability measure $\mu$ on $G$ to be a positive, unital, linear map from $C(G)$ into $\mathscr{C}$. Such a measure is completely positive and

$$
\|\mu\|=\|\mu(1)\|=1 \text {, }
$$

$[16, \S 4]$. Let $C(G, \mathscr{A}),(\cong C(G) \otimes \mathscr{A})$, denote the $C^{*}$-algebra of continuous functions from $G$ into $\mathscr{A}$, containing the algebraic tensor product $C(G) \odot \mathscr{A}$ as a dense *-subalgebra. The linear map

$$
C(G, \mathscr{A}) \ni X \rightarrow \hat{\mu}(X)=\int_{G} d \mu(g) X(g) \in \mathscr{A}
$$

is defined as follows. By [32, prop. 4.7] and nuclearity of $\mathscr{C}$, there is a unique *-homomorphism $\varepsilon$ of $\mathscr{C} \otimes \mathscr{A}$ onto $\mathscr{A}$ such that

$$
\varepsilon(c \otimes a)=c a, \quad c \in \mathscr{C}, a \in \mathscr{A} \text {. }
$$

Define $\hat{\mu}: C(G, \mathscr{A}) \rightarrow \mathscr{A}$ by

$$
\hat{\mu}=\varepsilon(\mu \otimes 1): C(G) \otimes \mathscr{A} \rightarrow \mathscr{C} \otimes \mathscr{A} \rightarrow \mathscr{A} .
$$

Then $\hat{\mu}$ is completely positive, and

$$
\hat{\mu}(f \otimes a)=\mu(f) a,
$$

for $f \in C(G)$ and $a \in \mathscr{A}$. We write $\int d \mu(g) X(g)$ for $\hat{\mu}(X)$ if $X \in C(G, \mathscr{A})$.

We need the following version of Bochner's theorem:

LEMMA 3.1. Let $G$ be a compact abelian group and $\mathscr{C}$ a unital abelian $C^{*}$-algebra, and $Z$ a function from $\hat{G}$ into $\mathscr{C}$ which is positive definite in the sense that the $n \times n$ matrix

$$
\left[Z\left(\gamma_{j}-\gamma_{i}\right)\right]_{i, j}
$$

is positive for all $\gamma_{1}, \ldots, \gamma_{n} \in \hat{G}, n \geq 1$, and $Z(0)=1$. Then there exists a $\mathscr{C}$-valued probability measure $\mu$ on $G$ such that

for all $\gamma$ in $\hat{G}$.

$$
Z(\gamma)=\mu(\langle\gamma, \cdot\rangle)=\int_{G} d \mu(g)\langle\gamma, g\rangle
$$


Proof. If $K$ is the spectrum of $\mathscr{C}$ and $\omega \in K$, then $\gamma \rightarrow \omega(Z(\gamma))$ is a positive definite function in the usual sense, and by Bochner's theorem there is a probability measure $\mu_{\omega}$ on $G$ with

If $h \in C(G)$, define

$$
Z(\gamma)(\omega)=\int_{G} d \mu_{\omega}(g)\langle\gamma, g\rangle
$$

$$
\tilde{h}(\omega)=\int_{G} d \mu_{\omega}(g) h(g)
$$

Since $h$ can be approximated uniformly by linear combinations of characters on $G$, it follows that $\tilde{h}$ is continuous on $K$, and so can be identified with an element $\mu(h)$, say, in $\mathscr{C}$. Clearly $\mu(1)=1$, and $\mu$ is a positive linear map from $C(G)$ into $\mathscr{C}$, and so is a $\mathscr{C}$-valued probability measure on $G$. Since

we have

$$
Z(\gamma)(\omega)=\int d \mu_{\omega}(g)\langle\gamma, g\rangle
$$

$$
Z(\gamma)=\mu(\langle\gamma, \cdot\rangle)
$$

Remark 3.2. If $\mu$ is a $\mathscr{C}$-valued probability measure on $G$, and $\omega \in K=$ Spectrum of $\mathscr{C}$, then an ordinary probabiity measure $\mu_{\omega}$ on $G$ is determined by

$$
\mu(h)(\omega)=\int_{G} d \mu_{\omega}(g) h(g),
$$

for all $h \in C(G)$. Thus $\mu$ determines a bundle $\mu_{\omega}$ of ordinary probability measures over $K$, and conversely the bundle $\left(\mu_{\omega}\right)$ determines $\mu$. Since we will use $\mathscr{C}$-valued probability measures only to formulate results, and not as a technical tool in the arguments, this bundle structure will not be emphasised except in the trivial case $\mathscr{C} \cong \mathbb{C}$.

\section{Completely positive maps}

In this section we consider the case of a single completely positive map commuting with a compact action. The following lemma is needed to make one of the hypotheses in theorem 4.2 meaningful (see [23, lemma 4.2] for a related result):

LEMMA 4.1. Let $G$ be a compact abelian group and $\tau$ an action of $G$ on a $C^{*}$-algebra $\mathscr{A}$. Any approximate identity for the fixed point algebra $\mathscr{A}^{\tau}$ is also an approximate identity for $\mathscr{A}$, and the multiplier algebra $M\left(\mathscr{A}^{\top}\right)$ is contained in $M(\mathscr{A})$.

Proof. Let $E_{\alpha}$ be an approximate identity for $\mathscr{A}^{+}$. If $X \in \mathscr{A}^{\top}(\gamma)$, then $X X^{*} \in \mathscr{A}^{\tau}$, and

$$
\begin{aligned}
\left(E_{\alpha} X-X\right)\left(E_{\alpha} X-X\right)^{*} & =E_{\alpha} X X^{*} E_{\alpha}-E_{\alpha} X X^{*}-X X^{*} E_{\alpha}+X X^{*} \\
& \rightarrow X X^{*}-X X^{*}-X X^{*}+X X^{*}=0
\end{aligned}
$$

as $\alpha \rightarrow \infty$. Thus

$$
E_{\alpha} X \rightarrow X
$$

for all $X$ in $\mathscr{A}_{F}$, and hence for all $X$ in $\mathscr{A}$. Let $Y \in M\left(\mathscr{A}^{\tau}\right)$ and $X \in \mathscr{A}$, then

$$
Y X=\lim _{\alpha} Y\left(E_{\alpha} X\right)=\lim _{\alpha}\left(\left(Y E_{\alpha}\right) X\right) \in \mathscr{A} \text {. }
$$

The second remark is now clear. 
THEOREM 4.2. Let $G$ be a compact abelian group and $\tau$ an action of $G$ on a $C^{*}$-algebra $\mathscr{A}$. Assume that the ideals $\mathscr{A}^{\tau}(\gamma) \mathscr{A}^{\top}(\gamma)^{*}$ in $\mathscr{A}^{\tau}$ are either dense or zero for each $\gamma$ in $\hat{G}$, and that the centre of $M\left(\mathscr{A}^{\tau}\right)$ is contained in the centre of $M(\mathscr{A})$. If $S: \mathscr{A} \rightarrow \mathscr{A}$ is a linear map, then the following two conditions are equivalent:

(4.1) (a) $S$ is completely positive;

(b) $S_{\tau}(g)=\tau(g) S, \quad$ for all $g$ in $G$;

(c) $S(X)=X, \quad$ for all $X$ in $\mathscr{A}^{\tau}$.

(4.2) There exists a centre $M\left(\mathscr{A}^{\tau}\right)$ )-valued probability measure $\mu$ on $G$ such that

$$
S(X)=\int d \mu(g) \tau(g)(X), \quad \text { for all } X \text { in } \mathscr{A}
$$

Proof. That (4.2) implies (4.1) is trivial. Suppose that (4.1) holds. Let $\mathscr{K}$ denote the Pedersen ideal of $\mathscr{A}^{\tau}$. If $\gamma_{1}$ and $\gamma_{2}$ are contained in the Arveson spectrum $\operatorname{Sp}(\tau)$ then

$$
\mathscr{K} \subseteq \mathscr{A}^{\tau}\left(\gamma_{i}\right)^{*} \mathscr{A}^{\tau}\left(\gamma_{i}\right) \quad \text { for } i=1,2
$$

since $\mathscr{K}$ is the minimal dense ideal in $\mathscr{A}^{\top}$ [26, theorem 5.6.1]. Thus

$$
\mathscr{K} \subseteq \mathscr{A}^{\tau}\left(\gamma_{1}\right)^{*} \mathscr{A}^{\tau}\left(\gamma_{1}\right) \mathscr{A}^{\tau}\left(\gamma_{2}\right)^{*} \mathscr{A}^{\tau}\left(\gamma_{2}\right),
$$

and so

$$
\mathscr{A}^{\tau}\left(\gamma_{1}\right) \mathscr{A}^{\tau}\left(\gamma_{2}\right)^{*} \neq\{0\} \quad \text { and } \quad \gamma_{1}-\gamma_{2} \in \operatorname{Sp}(\tau) \text {. }
$$

Thus $\operatorname{Sp}(\tau)$ is a subgroup of $\hat{G}$. By going to the quotient $G /(\operatorname{Sp}(\tau))^{\perp}$, we may assume that the action $\tau$ is faithful, and $\mathscr{A}^{\tau}(\gamma) \mathscr{A}^{\tau}(\gamma)^{*}$ is dense in $\mathscr{A}^{\tau}$ for all $\gamma$ in $G$.

LEMMA 4.3. If $S: \mathscr{A} \rightarrow \mathscr{A}$ is a linear map satisfying (4.1)(c), and the Kadison -Schwarz inequality $S\left(X^{*} X\right) \geq S(X)^{*} S(X)$ holds for all $X$ in $\mathscr{A}$, then

$$
S(X Y)=X S(Y) \quad S(Y X)=S(Y) X
$$

for all $X$ in $\mathscr{A}^{\tau}$ and $Y$ in $\mathscr{A}$.

Proof. Following [14], put

$$
D(X, Y)=\phi\left(S\left(X^{*} Y\right)-S(X)^{*} S(Y)\right),
$$

for $X$ and $Y$ in $\mathscr{A}$, where $\phi$ is a state on $\mathscr{A}$. Then $D$ is a non-negative sesqui-linear form. If $X \in \mathscr{A}^{\tau}$, then $X^{*}$ and $X X^{*} \in \mathscr{A}^{\tau}$, and (4.1)(c) implies that $D\left(X^{*}, X^{*}\right)=0$. Hence the Cauchy-Schwarz inequality, applied to $D$, gives

$$
D\left(X^{*}, Y\right)=0
$$

for all $X$ in $\mathscr{A}^{\tau}$ and $Y$ in $\mathscr{A}$. Since $S(X)=X$, for $X$ in $\mathscr{A}^{\tau}$, this implies $S(X Y)=$ $X S(Y)$.

Lemma 4.4. Assume that $\mathscr{A}^{\top}(\gamma) \mathscr{A}^{\top}(\gamma)^{*}$ is dense in $\mathscr{A}^{\tau}$ for all $\gamma$ in $\hat{G}$, and assume that $S: \mathscr{A} \rightarrow \mathscr{A}$ is a linear map satisfying (4.1). Then for each $\gamma$ in $\hat{G}$, there exists an element $Z(\gamma)$ in the centre of $M\left(\mathscr{A}^{\tau}\right)$ such that

$$
\boldsymbol{S}(\boldsymbol{X})=Z(\gamma) \boldsymbol{X}
$$

for all $X$ in $\mathscr{A}^{\tau}(\gamma)$. 
Proof. As $\mathscr{A}^{\tau}(\gamma) \mathscr{A}^{\tau}(\gamma)^{*}$ is a dense ideal in $\mathscr{A}^{\tau}$, it follows from [10] and lemma 4.1, or from [23, lemma 4.3], that $\mathscr{A}$ has an approximate identity of the form

$$
E_{\alpha}=\sum_{i} A_{i}^{\alpha} A_{i}^{\alpha *}
$$

where $A_{i}^{\alpha} \in \mathscr{A}^{\tau}(\gamma)$ and each sum is finite. Define

$$
Z_{\alpha}=\sum_{i} S\left(A_{i}^{\alpha}\right) A_{i}^{\alpha *}
$$

If $X \in \mathscr{A}^{\tau}(\gamma)$, we have by lemma 4.3

$$
S\left(E_{\alpha} X\right)=\sum_{i} S\left(A_{i}^{\alpha} A_{i}^{\alpha *} X\right)=\sum S\left(A_{i}^{\alpha}\right) A_{i}^{\alpha *} X=Z_{\alpha} X
$$

Hence

$$
S(X)=\lim _{\alpha} Z_{\alpha} X
$$

Next we derive a uniform estimate on $\left\|Z_{\alpha}\right\|$. As $S$ is positive we have by $(4.1)(c)$ that

$$
\|S\|=\lim \left\|S\left(E_{\alpha}\right)\right\|=\lim \left\|E_{\alpha}\right\|=1 .
$$

If $I_{n}$ is the identity map on $M_{n}=M_{n}(C)$, then it follows from complete positivity that $\left\|S \otimes I_{n}\right\|=1$.

Let

$$
A^{\alpha}=\left[\begin{array}{ccc}
A_{1}^{\alpha} & \ldots & A_{n}^{\alpha} \\
0 & \ldots & 0 \\
\vdots & & \vdots \\
0 & \ldots & 0
\end{array}\right]
$$

Then

$$
A^{\alpha} A^{\alpha *}=\left[\begin{array}{cccc}
E_{\alpha} & 0 & \ldots & 0 \\
0 & & & \\
\vdots & & & \vdots \\
0 & 0 & \ldots & 0
\end{array}\right], \quad\left(S \otimes I_{n}\right)\left(A^{\alpha}\right) A^{\alpha *}=\left[\begin{array}{cccc}
Z_{\alpha} & 0 & \ldots & 0 \\
0 & & & 0 \\
\vdots & & & \vdots \\
0 & 0 & \ldots & 0
\end{array}\right]
$$

In particular, $\left\|A^{\alpha}\right\| \leq\left\|E_{\alpha}\right\|^{\frac{1}{2}} \leq 1$, and so

$$
\left\|Z_{\alpha}\right\| \leq\left\|S \otimes I_{n}\right\|\left\|A^{\alpha}\right\|^{2} \leq 1
$$

This is the desired uniform estimate. We now show that $Z_{\alpha}$ converges in the strict topology on $M\left(\mathscr{A}^{\tau}\right)$ to an element $Z(\gamma)$ in $M\left(\mathscr{A}^{\tau}\right)$ (i.e. $Z_{\alpha} X \rightarrow Z(\gamma) X$ and $X Z_{\alpha} \rightarrow$ $X Z(\gamma)$ in norm for each $\left.X \in \mathscr{A}^{\tau}\right)$. First, we have

$$
Z_{\alpha} E_{\beta}=\sum_{i} Z_{\alpha} A_{i}^{\beta} A_{i}^{\beta *} \rightarrow \sum S\left(A_{i}^{\beta}\right) A_{i}^{\beta *}, \quad \text { as } \alpha \rightarrow \infty .
$$

Since $E_{\beta}$ forms an approximate identity for $\mathscr{A}$, and $\left\|Z_{\alpha}\right\| \leq 1$, it follows that $\lim _{\alpha} Z_{\alpha} Y$ exists for all $Y$ in $\mathscr{A}$, and the limit has norm not greater than $\|Y\|$. Thus $Z_{\alpha}$ converges strongly to an element $Z(\gamma)$ in the universal representation of $\mathscr{A}$. Hence $Z(\gamma) \in \mathscr{A}^{* *}$, the bidual of $\mathscr{A}$, and

$$
Z(\gamma) Y=\lim _{\alpha} Z_{\alpha} Y \in \mathscr{A}, \quad \text { for all } Y \text { in } \mathscr{A}
$$

i.e. $Z(\gamma)$ is a left multiplier of $\mathscr{A}$. Also

$$
S(X)=\lim Z_{\alpha} X=Z(\gamma) X, \quad \text { for all } X \text { in } \mathscr{A}^{\tau}(\gamma) .
$$


If $X \in \mathscr{A}^{\tau}(\gamma)$ and $Y \in \mathscr{A}^{\tau}$ then $Y X \in \mathscr{A}^{\tau}(\gamma)$, and so $S(Y X)=Z(\gamma) Y X$. But lemma 4.3 shows that

$$
S(Y X)=Y S(X)=Y Z(\gamma) X
$$

and hence

$$
Z(\gamma) Y X=Y Z(\gamma) X
$$

But then

$$
\begin{aligned}
Z(\gamma) Y E_{\alpha} & =\sum_{i}\left(Z(\gamma) Y A_{i}^{\alpha}\right) A_{i}^{\alpha *} \\
& =\sum_{i}\left(Y Z(\gamma) A_{i}^{\alpha}\right) A_{i}^{\alpha *} \\
& =Y Z(\gamma) E_{\alpha},
\end{aligned}
$$

and as $E_{\alpha}$ converges strongly to 1 in the universal representation we get

$$
Z(\gamma) Y=Y Z(\gamma),
$$

for all $Y$ in $\mathscr{A}^{\tau}$. This shows $Z(\gamma) \in \mathscr{A}^{\tau \prime}$, and since $Z(\gamma)$ is a left multiplier,

$$
X E_{\alpha} Z(\gamma)=X Z(\gamma) E_{\alpha} \in \mathscr{A}
$$

for all $\alpha$. But then

$$
X Z(\gamma)=\lim _{\alpha} X E_{\alpha} Z(\gamma) \in \mathscr{A}
$$

and so $Z(\gamma)$ is also a right multiplier.

LEMMA 4.5. Adopt the assumptions of lemma 4.4, and also assume that the centre of $M\left(\mathscr{A}^{\tau}\right)$ is contained in the centre of $M(\mathscr{A})$. Then

$$
\gamma \in \hat{G} \rightarrow Z(\gamma) \in \text { centre } M\left(\mathscr{A}^{\tau}\right)
$$

is positive definite.

Proof. Take $X_{i} \in \mathscr{A}^{\tau}\left(\gamma_{i}\right)$ for $i=1, \ldots, n$. Then the $n \times n$ matrix $\left[S\left(X_{i}^{*} X_{j}\right)\right]$ is positive since $S$ is completely positive. But

$$
\boldsymbol{X}_{i}^{*} \boldsymbol{X}_{j} \in \mathscr{A}^{\tau}\left(-\gamma_{i}\right) \mathscr{A}^{\tau}\left(\gamma_{j}\right) \subseteq \mathscr{A}^{\tau}\left(\gamma_{j}-\gamma_{i}\right)
$$

and so

$$
S\left(X_{i}^{*} X_{j}\right)=Z\left(\gamma_{i}-\gamma_{i}\right) X_{i}^{*} X_{j}=X_{i}^{*} Z\left(\gamma_{j}-\gamma_{i}\right) X_{j}
$$

and hence

$$
\left[X_{i}^{*} Z\left(\gamma_{j}-\gamma_{i}\right) X_{j}\right] \geq 0
$$

But then

$$
\left[\begin{array}{cccc}
\boldsymbol{X}_{1} & 0 & \ldots & 0 \\
0 & \boldsymbol{X}_{2} & \ldots & 0 \\
\vdots & & & \vdots \\
0 & \ldots & \ldots & \boldsymbol{X}_{n}
\end{array}\right]\left[\boldsymbol{X}_{i}^{*} \boldsymbol{Z}\left(\gamma_{j}-\gamma_{i}\right) \boldsymbol{X}_{j}\right]\left[\begin{array}{cccc}
\boldsymbol{X}_{1}^{*} & 0 & \ldots & 0 \\
0 & \boldsymbol{X}_{2}^{*} & \ldots & 0 \\
\vdots & & & \vdots \\
0 & \ldots & \ldots & \boldsymbol{X}_{n}^{*}
\end{array}\right] \geq 0
$$

i.e.

$$
\left[X_{i} X_{i}^{*} Z\left(\gamma_{j}-\gamma_{i}\right) X_{i} X_{i}^{*}\right] \geq 0
$$


We next show that if $X_{i}^{k} \in \mathscr{A}^{\top}\left(\gamma_{i}\right)$, for $i=1, \ldots, n$, and $k=1, \ldots, m$, then the $n \times n$ matrix

is positive. Let

$$
\left[\left(\sum_{k} X_{i}^{k} X_{i}^{k *}\right) Z\left(\gamma_{j}-\gamma_{i}\right)\left(\sum_{k} X_{j}^{k} X_{j}^{k *}\right)\right]
$$

$$
\tilde{A}=\mathscr{A} \otimes M_{m}, \quad \tilde{S}=S \otimes I_{m}, \quad \tilde{Z}(\gamma)=Z(\gamma) \otimes 1_{m}, \quad \tilde{\tau}_{\mathrm{g}}=\tau_{\mathrm{g}} \otimes \iota .
$$

Then one easily verifies that

$$
\tilde{\mathscr{A}}^{\tilde{\tau}}(\gamma)=\mathscr{A}^{\tau}(\gamma) \otimes M_{m},
$$

and hence that $\tilde{S}(\tilde{X})=\tilde{Z}(\gamma) \tilde{X}$ for all $\tilde{X} \in \tilde{\mathcal{A}}^{\tilde{\gamma}}(\gamma)$. Furthermore $\tilde{Z}(\gamma)$ is contained in the centre of $M(\tilde{\mathscr{A}})=M(\mathscr{A}) \otimes M_{m}$, and $\tilde{S}$ is completely positive. The above reasoning applied to $\tilde{S}$ then implies that for $\tilde{X}_{i} \in \mathscr{A}^{\tau}\left(\gamma_{i}\right)=\mathscr{A}^{\tau}\left(\gamma_{i}\right) \otimes M_{m}, i=1, \ldots, n$ we have

$$
\left[\tilde{X}_{i} \tilde{X}_{i}^{*} \tilde{Z}\left(\gamma_{j}-\gamma_{i}\right) \tilde{X}_{j} \tilde{X}_{i}^{*}\right] \geq 0, \quad \text { in } M_{m n}(\mathscr{A}) .
$$

Now make the special selection

$$
\tilde{X}_{i}=\left[\begin{array}{cccc}
X_{i}^{1} & X_{i}^{2} & \ldots & X_{i}^{m} \\
0 & 0 & \ldots & 0 \\
\vdots & & & \vdots \\
0 & 0 & \ldots & 0
\end{array}\right], \text { where } X_{i}^{k} \in \mathscr{A}^{\top}\left(\gamma_{i}\right)
$$

Then

$$
\tilde{X}_{i} \tilde{X}_{i}^{*} \tilde{Z}\left(\gamma_{j}-\gamma_{i}\right) \tilde{X}_{j} \tilde{X}_{j}^{*}=\left[\begin{array}{cccc}
\left(\sum_{k} X_{i}^{k} X_{i}^{k *}\right) Z\left(\gamma_{j}-\gamma_{i}\right)\left(\sum_{k} X_{j}^{k} X_{j}^{k *}\right) & 0 & \ldots & 0 \\
0 & 0 & & 0 \\
\vdots & \vdots & & \vdots \\
0 & 0 & \ldots & 0
\end{array}\right]
$$

and going to a submatrix, this implies

$$
\left[\sum_{k}\left(X_{i}^{k} X_{i}^{k *}\right) Z\left(\gamma_{j}-\gamma_{i}\right)\left(\sum_{k} X_{i}^{k} X_{j}^{k *}\right)\right] \geq 0 .
$$

As in the proof of lemma 4.4 , we can find an approximate identity for $\mathscr{A}$ consisting of elements of the form $\sum_{k} X_{i}^{k} X_{i}^{k *}$, where $X_{i}^{k} \in \mathscr{A}^{\tau}\left(\gamma_{i}\right)$, which converge strongly to 1 in the universal representation. Hence we get from the above relation that

$$
\left[Z\left(\gamma_{i}-\gamma_{i}\right)\right] \geq 0 \text {. }
$$

End of proof of theorem 4.2. By lemmas 4.4 and 4.5, there exists a positive definite map $\gamma \in \hat{G} \rightarrow Z(\gamma) \in$ centre $M\left(\mathscr{A}^{\tau}\right)$ such that $S(X)=Z(\gamma) X$, for $X$ in $\mathscr{A}^{\tau}(\gamma)$. By lemma 3.1, there exists a centre $\left(M\left(\mathscr{A}^{\tau}\right)\right)$-valued probability measure $\mu$ on $G$ such that

$$
Z(\gamma)=\mu(\langle\gamma, \cdot\rangle)
$$

If $\int d \mu(g)$ is the associated positive map from $C(G, \mathscr{A})$ into $\mathscr{A}$, we have for $X \in \mathscr{A}^{\top}(\gamma)$ :

$$
\begin{aligned}
\int d \mu(g) \tau(g)(X) & =\int d \mu(g)\langle\gamma, g) X \\
& =\mu((\gamma, \cdot\rangle) X \\
& =Z(\gamma) X=S(X) .
\end{aligned}
$$


Hence by linearity $S(X)=\int_{G} d \mu(g) \tau(g)(X)$, for all $X$ in $\mathscr{A}_{F}$, and hence for all $X$ in $\mathscr{A}$ by continuity.

COROLlARy 4.6. Let $G$ be a compact abelian group and $\tau$ an action of $G$ on a $C^{*}$-algebra $\mathscr{A}$ such that $\mathscr{A}^{\tau}$ is simple. If $S: \mathscr{A} \rightarrow \mathscr{A}$ is a linear map, then the following two conditions are equivalent:

(4.3) (a) $S$ is completely positive;

(b) $S \tau(g)=\tau(g) S, \quad$ for all $g$ in $G$;

(c) $S(X)=X, \quad$ for all $X$ in $\mathscr{A}^{\tau}$.

(4.4) There exists a probability measure $\mu$ on $G$ such that

$$
S(X)=\int d \mu(g) \tau(g) X, \quad \text { for all } X \text { in } \mathscr{A} .
$$

Proof. When $\mathscr{A}^{\tau}$ is simple, the ideals $\mathscr{A}^{\tau}(\gamma) \mathscr{A}^{\tau}(\gamma)^{*}$ are either zero or dense. The Dauns Hofmann theorem states that the centre of $M\left(\mathscr{A}^{\tau}\right)$ is isomorphic to the $C^{*}$-algebra $C_{b}\left(\operatorname{Prim} \mathscr{A}^{\tau}\right)$ of all continuous bounded functions on the primitive ideal space of $\mathscr{A}^{\tau}$, and hence the centre of $M\left(\mathscr{A}^{\tau}\right)$ is trivial, when $\mathscr{A}^{\tau}$ is simple [26]. Thus theorem 4.2 applies and $\mu$ is an ordinary probability measure on $G$ in this case.

We next consider a single completely positive map commuting with a compact action on a von Neumann algebra.

Proposition 4.7. Let $G$ be a compact abelian group and $\tau$ an action of $G$ on a von Neumann algebra $\mathcal{M}$. Let $S: M \rightarrow \mathcal{M}$ be a linear map such that

(a) $S$ is completely positive;

(b) $S \tau(g)=\tau(g) S$, for all $g$ in $G$;

(c) $S(X)=X, \quad$ for all $X$ in $\mathcal{M}^{\tau}$.

Then $S$ is normal.

Proof. The linear space $\mathscr{M}^{\tau}(\gamma) \mathcal{M}^{\tau}(\gamma)^{*}$ is an ideal in $\mathscr{M}^{\tau}$, and hence there exists a projection $E(\gamma)$ in $\mathscr{M}^{\tau} \cap \mathcal{M}^{\tau \prime}$ such that $\overline{\mathcal{M}^{\tau}(\gamma) \mathcal{M}^{\tau}(\gamma)^{*}}=\mathcal{M}^{\tau} E(\gamma)$, where the bar denotes $\sigma$-weak closure. By minor modifications to the proof of lemma 4.4 we can produce an element

$$
Z(\gamma) \in \operatorname{centre}\left(\mathscr{M}^{\tau} E(\gamma)\right)=\left(\mathscr{M}^{\tau} \cap \mathscr{M}^{\tau \prime}\right) E(\gamma)
$$

such that $S(X)=Z(\gamma) X$ for all $X$ in $\mathscr{M}^{\gamma}(\gamma)$.

The expression on the right makes sense since the range of $X$ is contained in $E(\gamma)$, and we may extend $Z(\gamma)$ to an operator in $\mathscr{M}^{\tau} \cap \mathcal{M}^{\tau \prime}$ by requiring

$$
Z(\gamma)(1-E(\gamma))=0 .
$$

The identity $S(X)=Z(\gamma) X$ is then still valid. If $X \in \mathcal{M}$, we let

$$
\{X(\gamma)\}_{\gamma \in \hat{G}},
$$

denote its Fourier components relative to $\tau$, i.e.

$$
X(\gamma)=P_{\gamma} X .
$$


Let $\tau_{*}$ be the dual action of $\tau$ on $\mathscr{M}_{*}$. The convex set of $G$-finite elements $\mathscr{M}_{* F}$ for the action is weakly dense, hence norm dense in $\mathcal{M}_{*}$. If $\eta \in \mathcal{M}_{*}^{\tau}(\gamma)$ then

$$
\eta(X)=\eta(X(\gamma)) \quad \text { for all } X \text { in } \mathcal{M} .
$$

Since $S$ commutes with $\tau$ one deduces further that

$$
\eta(S(X))=\eta(S(X(\gamma)))=\eta(Z(\gamma) X(\gamma))={ }_{z(\gamma)} \eta(X(\gamma))={ }_{z_{(\gamma},} \eta(X)
$$

where $Z(\gamma) \eta$ is the left translate of $\eta$ by $Z(\gamma)$, and the last identity follows from the fact that $Z(\gamma) \in \mathscr{M}^{\top}$. Hence

$$
S^{*} \eta={ }_{z(\gamma)} \eta
$$

and as $Z_{(\gamma)} \eta \in \mathscr{M}_{*}$ it follows that

$$
S^{*}\left(\mathcal{M}_{* F}\right) \subseteq \mathscr{M}_{* F} \subseteq \mathscr{M}_{*}
$$

But $\mathscr{M}_{* F}$ is norm dense in $\mathcal{M}_{*}$, so

$$
S^{*} \mathcal{M}_{*} \subseteq \mathscr{M}_{*}
$$

and hence $S$ is normal.

We are now ready to state a von Neumann version of theorem 4.2.

COROLlary 4.8. Let $G$ be a compact abelian group and $\tau$ an action of $G$ on a von Neumann algebra $\mathcal{M}$ such that the ideals $\mathscr{M}^{\top}(\gamma) \mathcal{M}^{\top}(\gamma)^{*}$ in $\mathscr{M}^{\tau}$ are either zero or $\sigma$-weakly dense, and $\mathscr{M}^{\tau} \cap \mathcal{M}^{\tau \prime}$ is contained in $\mathscr{M} \cap \mathscr{M}^{\prime}$. Then there is a bijection between:

(4.5) Linear maps $S: M \rightarrow M$ such that

(a) $S$ is completely positive;

(b) $S \tau(g)=\tau(g) S \quad$ for all $g$ in $G$;

(c) $S(X)=X \quad$ for all $X$ in $\mathcal{M}^{\tau}$;

and

(4.6) $\left(\mathcal{M}^{\top} \cap \mathcal{M}^{\top \prime}\right)$-valued measures $\mu$ on $G$.

The correspondence is given by

$$
S(X)=\int_{G} d \mu(g) \tau(g)(X)
$$

for all $X$ in the strong continuity subspace $\mathcal{M}_{0}$ for $\tau$.

Remark 4.9. The two conditions

(a) $\mathscr{M}^{\tau}(\gamma) \mathscr{M}^{\tau}(\gamma)^{*}$ is either dense in $\mathcal{M}^{\tau}$ or zero for each $\gamma \in \hat{G}$,

(b) $\mathscr{M}^{\tau} \cap \mathscr{M}^{\tau \prime} \subseteq M \mathcal{M}^{\prime} \cap \mathcal{M}^{\prime}$,

are equivalent to the single condition

(ab) $\Gamma(\tau)=\operatorname{Sp}(\tau)$,

where $\Gamma(\tau)$ denotes the $\Gamma$-spectrum of $\tau$ and $\operatorname{Sp}(\tau)$ is the spectrum of $\tau$ (at least when $\mathcal{M}_{*}$ is separable). We give a crude outline of the argument.

Assume first that $\tau$ is centrally ergodic, i.e.

$$
\mathscr{M}^{\top} \cap\left(\mathcal{M} \cap \mathcal{M}^{\prime}\right)=\mathbb{C} 1 .
$$


This is equivalent to the fact that $\mathcal{M}$ has no non-trivial $\sigma$-weakly closed $\tau$-invariant ideals. In this situation one has that $\mathcal{M}^{\tau}$ is a factor if and only if $\Gamma(\tau)=\operatorname{Sp}(\tau),[\mathbf{2 6}$, Proof of theorem 8.10.4.], i.e.

$$
\mathscr{M}^{\top} \cap \mathcal{M}^{\tau \prime} \subseteq \mathcal{M} \cap \mathscr{M}^{\prime}
$$

if and only if $\Gamma(\tau)=\mathrm{Sp}(\tau)$.

In the general case, decompose $\mathscr{M}$ over the abelian von Neumann sub-algebra $\mathscr{M}^{\tau} \cap\left(\mathscr{M} \cap \mathscr{M}^{\prime}\right)$. As

$$
\mathscr{M}^{\tau} \cap \mathcal{M}^{\tau \prime} \subseteq \mathscr{M} \cap \mathscr{M}^{\prime}
$$

if and only if

$$
\mathscr{M}^{\tau} \cap \mathscr{M}^{\tau \prime} \subseteq \mathscr{M}^{\tau} \cap\left(\mathcal{M} \cap \mathscr{M}^{\prime}\right),
$$

it follows from the above that this condition holds if and only if $\Gamma=\mathrm{Sp}$ on each of the minimal components of the decomposition. But condition (a) ensures that the spectrum is constant on almost all components, and hence (a) and (b) imply (ab). Conversely $(\mathrm{ab})$ implies that

$$
\Gamma=\operatorname{Sp}=\operatorname{Sp}(\tau)
$$

on almost all components, and hence (b) holds. But if $\Gamma(\tau)=\operatorname{Sp}(\tau)$ and $\gamma \in \operatorname{Sp}(\tau)$, and $E \in \mathscr{M}^{\tau}$ is a projection, then $\gamma \in \Gamma(\tau)$ and hence $E \mathscr{M}^{\tau}(\gamma) E \neq\{0\}$, thus

$$
E \mathscr{M}^{\tau}(\gamma) \mathscr{M}^{\tau}(\gamma)^{*} E \supseteq E \mathscr{M}^{\tau}(\gamma) E \mathscr{M}^{\tau}(\gamma)^{*} E \neq\{0\}
$$

and thus $\mathcal{M}^{\tau}(\gamma) \mathcal{M}^{\tau}(\gamma)^{*}$ is dense in $\mathcal{M}^{\tau}$, i.e. (a) holds. This shows (a) \& (b) $\Leftrightarrow$ (ab).

Proof of corollary 4.8. If $S$ satisfies (4.5), then it is normal by proposition 4.7. By (4.5)(b), $S$ maps the $C^{*}$-algebra $\mathcal{M}_{0}$, the strong continuity subalgebra, into itself, and theorem 4.2 applies to show that $\left.S\right|_{\mu_{0}}$ is given by

$$
S(X)=\int d \mu(g) \tau(g)(X),
$$

where $\mu$ is an $\mathscr{M}^{\top} \cap \mathcal{M}^{\tau \prime}$-valued probability measure. (Actually the ideals $\mathscr{M}^{\tau}(\gamma) \mathscr{M}^{\tau}(\gamma)^{*}$ are only $\sigma$-weakly dense in $\mathscr{M}^{\tau}$, but by modifying the proof of theorem 4.2 as indicated in the proof of proposition 4.7 , one can produce the elements $Z(\gamma)$ as before and use lemma 3.1 to construct the measure $\mu$.) Since $S$ is normal, it is uniquely determined by its restriction to the $\sigma$-weakly dense subalgebra $\mathcal{M}_{0}$.

Conversely, if $\mu$ is given, it defines a completely positive map $S$ on $\mathcal{M}_{0}$ by

$$
S(X)=\int d \mu(g) \tau(g)(X) .
$$

If $X \in \mathscr{M}^{\top}(\gamma)$ one has

$$
S(X)=Z(\gamma) X
$$

where

$$
Z(\gamma)=\mu(\langle\gamma, \cdot\rangle) \in \mathscr{M}^{\tau} \cap \mathcal{M}^{\tau \prime} .
$$

Using this, as in the proof of proposition 4.7 , it follows that $S$ extends by $\sigma$-weak continuity to $\mathscr{M}$.

The hypothesis of the following corollary is often satisfied when $\mathscr{A}^{\tau}$ is not simple but prime. 
COROLlARY 4.10. Let $G$ be a compact abelian group and $\tau$ an action of $G$ on $a$ $C^{*}$-algebra $\mathscr{A}$. Let

$$
P(X)=\int d g \tau(g)(X)
$$

be the canonical projection of $\mathscr{A}$ on $\mathscr{A}^{\top}$. Assume that there exists a faithful representation $\pi_{0}$ of $\mathscr{A}^{\tau}$ such that $\pi\left(\mathscr{A}^{\tau}\right)^{\prime \prime}$ is a factor, where $\pi$ is the Stinespring representation associated to the completely positive map $\pi_{0}^{\circ} P$. If $S: \mathscr{A} \rightarrow \mathscr{A}$ is a linear map, then the following two conditions are equivalent:

(4.7) (a) $S$ is completely positive;

(b) $S \tau(g)=\tau(g) S$ for all $g$ in $G$;

(c) $S(X)=X \quad$ for all $X$ in $\mathscr{A}^{\tau}$.

(4.8) There exists a probability measure $\mu$ on $G$ such that

$$
S(X)=\int d \mu(g) \tau(g)(X) \text { for all } X \text { in } \mathscr{A} \text {. }
$$

Remark 4.11. The assumptions on the dynamics in this corollary imply that $\mathscr{A}^{\tau}$ is prime. Conversely, if $\mathscr{A}^{\tau}$ is prime, are the assumptions fulfilled? If $\mathscr{A}$ is $G$-prime, then $\mathscr{A}^{\tau}$ being prime is equivalent to $\Gamma(\tau)=\mathrm{Sp}(\tau),([26$, theorem 8.10 .4$])$, and the problem would have a positive solution if $\mathscr{A}$ has a faithful $G$-invariant factor representation with $\Gamma(\hat{\tau})=\Gamma(\tau)$, where $\hat{\tau}$ is the extension of $\tau$ to the weak closure in this representation.

Proof. Suppose (4.7) holds, and assume that $\mathscr{A}$ is realised in the Stinespring representation. Since the completely positive map $\pi_{0}{ }^{\circ} P$ is $G$-invariant, there is a canonical strongly continuous unitary representation $U$ of $G$ such that

$$
\tau(g)(X)=U(g) X U(g)^{*}
$$

for all $X$ in $\mathscr{A}$. Then $\tau$ extends to an action of $G$ on the von Neumann algebra $\mathscr{M}=\mathscr{A}^{\prime \prime}$. Moreover,

$$
P(X)=\int_{G} d g \tau(g)(X)
$$

defines a $\sigma$-weakly continuous projection from $\mathcal{M}$ onto the minimal spectral subspace $\mathscr{M}^{\tau}$, and using this projection, it follows that $\mathscr{A}^{\tau}$ is $\sigma$-weakly dense in $\mathcal{M}^{+}$, or $\mathscr{M}^{\tau}=\left(\boldsymbol{A}^{\tau}\right)^{\prime \prime}$. In particular $\mathscr{M}^{\tau}$ is a factor. The map $S$ extends by $\sigma$-weak continuity to a completely positive map of $\mathscr{M}$. This follows from the proof of proposition 4.7 , or more directly as follows: as the projection $P: \mathcal{M} \rightarrow \mathcal{M}^{\top}$ is faithful, the subspace $\mathscr{K}$ of the representation Hilbert space $\mathscr{H}$ of $\mathscr{M}$ corresponding to $\pi_{0} \circ P$ is separating for $\mathscr{M}$ and hence cyclic for $\mathscr{M}^{\prime}$. Thus any positive normal linear functional on $\mathscr{M}$ can be approximated in norm by linear combinations of functionals of the form

$$
Y \in \mathscr{M} \rightarrow\left(\xi, X^{* *} Y X^{\prime} \xi\right)
$$

where $\xi \in \mathscr{K}, X^{\prime} \in \mathscr{M}^{\prime}$. But if $Y \geq 0$ we have

$$
\begin{aligned}
\left(\xi, X^{\prime *} S(Y) X^{\prime} \xi\right) & \leq\left\|X^{\prime}\right\|^{2}(\xi, S(Y) \xi) \\
& =\left\|X^{\prime}\right\|^{2}(\xi, P S(Y) \xi) \\
& =\left\|X^{\prime}\right\|^{2}(\xi, P(Y) \xi) \\
& =\left\|X^{\prime}\right\|^{2}(\xi, Y \xi),
\end{aligned}
$$


and hence $Y \rightarrow\left(\xi, X^{\prime *} S(Y) X^{\prime} \xi\right)$ is normal. It follows that the transpose of $S$ maps $\pi$-normal states into $\pi$-normal states and hence $S$ extends to $\mathcal{M}$ by $\sigma$-weak continuity. Applying corollary 4.8 on this extended $S$, corollary 4.10 is immediate, because the ideals $\mathscr{M}^{\tau}(\gamma) \mathscr{M}^{\tau}(\gamma)^{*}$ in $\mathscr{M}^{\tau}$ are either zero or $\sigma$-weakly dense as $\mathscr{M}^{\tau}$ is a factor. The factoriality of $\mathscr{M}^{\tau}$ also implies that the measure $\mu$ is an ordinary probability measure.

The converse is trivial.

The prototype for the following can be found in [15] and [1], [29], [33].

Proposition 4.12. Let $\mathcal{M}$ be a von Neumann algebra and $\mathcal{N}$ a von Neumann subalgebra of $\mathcal{M}$ with the same identities. Let $P: M \rightarrow N$ be a normal faithful projection of $\mathcal{M}$ onto $\mathcal{N}$, and $T: \mathcal{M} \rightarrow \mathcal{M}$ a completely positive normal map such that

$$
P T=P \text {. }
$$

Assume that $\mathcal{N}$ is realised on a Hilbert space $\mathscr{K}$, and $(\pi, \mathscr{H}, V)$ the Stinespring decomposition of

$$
P: \mathcal{M} \rightarrow B(\mathscr{K})
$$

Then there exists an unique bounded operator $W$ on $\mathscr{H}$ such that

$$
W \pi(X) V=\pi(T(X)) V \quad \text { for all } X \in \mathcal{M},
$$

and the map

$$
X \rightarrow \pi(T(X))-W \pi(X) W^{*}
$$

from $\mathscr{M}$ into $B(\mathscr{H})$ is completely positive.

Proof. $W$ is well defined as a contraction on $\mathscr{H}$ because

$$
\begin{aligned}
\left\|\sum_{i} \pi\left(T\left(X_{i}\right)\right) V \xi_{i}\right\|^{2} & =\sum_{i, j}\left\langle V \xi_{i}, \pi\left(T\left(X_{i}^{*}\right) T\left(X_{j}\right)\right) V \xi_{j}\right\rangle \\
& \leq \sum_{i, j}\left\langle V \xi_{i}, \pi\left(T\left(X_{i}^{*} X_{j}\right)\right) V \xi_{j}\right\rangle \\
& =\sum_{i, j}\left\langle\xi_{i}, V^{*} \pi\left(T\left(X_{i}^{*} X_{j}\right)\right) V \xi_{j}\right\rangle \\
& =\sum_{i, j}\left\langle\xi_{i}, P T\left(X_{i}^{*} X_{i}\right) \xi_{j}\right\rangle \\
& =\sum_{i, j}\left\langle\xi_{i}, P\left(X_{i}^{*} X_{j}\right) \xi_{j}\right\rangle \\
& =\left\|\sum \pi\left(X_{i}\right) V \xi_{i}\right\|^{2}
\end{aligned}
$$

for any $X_{i}$ in $\mathscr{M}, \xi_{i}$ in $\mathscr{K}$. Next, to any $Y^{\prime}$ in $\pi(\mathscr{M})^{\prime}$ we shall associate an element $T^{\prime}\left(Y^{\prime}\right)$ in $\pi(\mathscr{M})^{\prime}$ with the property that

$$
\left\langle V \xi, \pi(T(X)) Y^{\prime} V \eta\right\rangle=\left\langle V \xi, \pi(X) T^{\prime}\left(Y^{\prime}\right) V \eta\right\rangle
$$

for all $X$ in $\mathcal{M}, \xi$ and $\eta$ in $\mathscr{K}$. Assume first that $Y^{\prime} \geq 0$. 
If $\xi_{1}, \ldots, \xi_{n} \in \mathscr{K}, X_{1}, \ldots, X_{n} \in \mathcal{M}$ we have

$$
\begin{aligned}
0 & \leq \sum_{i, j}\left\langle Y^{\prime \frac{1}{2}} V \xi_{i}, \pi\left(T\left(X_{i}^{*} X_{j}\right)\right) Y^{\prime \frac{1}{2}} V \xi_{j}\right\rangle \\
& =\sum_{i, j}\left\langle V \xi_{i}, \pi\left(T\left(X_{i}^{*} X_{j}\right)\right) Y^{\prime} V \xi_{j}\right\rangle \\
& \leq\left\|Y^{\prime}\right\| \sum_{i, j}\left\langle V \xi_{i}, \pi\left(T\left(X_{i}^{*} X_{j}\right)\right) V \xi_{j}\right\rangle \\
& =\left\|Y^{\prime}\right\| \sum_{i, j}\left\langle\xi_{i}, P\left(X_{i}^{*} X_{j}\right) \xi_{j}\right\rangle,
\end{aligned}
$$

as before. If $\phi, \psi$ are linear maps between $C^{*}$-algebras, we write

$$
\phi \ll \psi
$$

if $\psi-\phi$ is completely positive. Then the above shows that

$$
0 \ll V^{*} \pi(T(\cdot)) Y^{\prime} V \ll\left\|Y^{\prime}\right\| P(\cdot)=\left\|Y^{\prime}\right\| V^{*} \pi(\cdot) V .
$$

By [3, theorem 1.4.2], there exists a $T^{\prime}\left(Y^{\prime}\right)$ in $\pi(\mathcal{M})^{\prime}$ such that

$$
V^{*} \pi(T(\cdot)) Y^{\prime} V=V^{*} \pi(\cdot) T^{\prime}\left(Y^{\prime}\right) V
$$

In fact, $T^{\prime}\left(Y^{\prime}\right)$ is the operator on $\mathscr{H}$ such that

$$
\sum_{i j}\left(V \xi_{i}, \pi\left(X_{i}^{*} Z_{j}\right) Y^{\prime} V \eta_{j}\right)=\left(\sum_{i} \pi\left(X_{i}\right) V \xi_{i}, T^{\prime}\left(Y^{\prime}\right) \sum_{j} \pi\left(Z_{j}\right) V \eta_{j}\right),
$$

and existence and uniqueness follows from Riesz's representation theorem.

By linearity, there exists for all $Y^{\prime}$ in $\pi(\mathcal{M})^{\prime}$ an unique operator $T^{\prime}\left(Y^{\prime}\right)$ in $\pi(\mathcal{M})^{\prime}$ such that

$$
\left\langle V \xi, \pi(T(X)) Y^{\prime} V \eta\right\rangle=\left\langle V \xi, \pi(X) T^{\prime}\left(Y^{\prime}\right) V \eta\right\rangle,
$$

for all $X$ in $\mathscr{M}, \xi$ and $\eta$ in $\mathscr{K} . T^{\prime}$ is clearly positive, and by a matrix argument, it is completely positive. The subspace $V \mathscr{K}$ is separating for $\pi(\mathcal{M})$ since $P$ is faithful, and so it is cyclic (and separating) for $\pi(\mathcal{M})^{\prime}$. Thus as in the first part of the proof, a contraction $W^{\prime}$ on $\mathscr{H}$ is uniquely defined by the requirement

$$
W^{\prime} Y^{\prime} V=T^{\prime}\left(Y^{\prime}\right) V \quad \text { for all } Y^{\prime} \text { in } \pi(M)^{\prime} \text {. }
$$

Then

$$
\begin{aligned}
\left\langle W \pi(X) V \xi, Y^{\prime} V \eta\right\rangle & =\left\langle\pi(T(X)) V \xi, Y^{\prime} V \eta\right\rangle \\
& =\left\langle\pi(X) V \xi, T^{\prime}\left(Y^{\prime}\right) V \eta\right\rangle=\left\langle\pi(X) V \xi, W^{\prime} Y^{\prime} V \eta\right\rangle
\end{aligned}
$$

for all $X$ in $\mathcal{M}, Y^{\prime}$ in $\pi(\mathcal{M})^{\prime}$ and $\xi, \eta$ in $\mathscr{K}$. Hence $W^{*}=W^{\prime}$. This shows finally that

$$
\begin{aligned}
& \sum_{i j k l}\left\langle Y_{i j}^{\prime} V \xi_{i j}, \pi\left(T\left(X_{i}^{*} X_{k}\right)\right) Y_{k l}^{\prime} V \xi_{k l}\right\rangle \\
= & \sum_{i j k l}\left\langle V \xi_{i j}, \pi\left(T\left(X_{i}^{*} X_{k}\right)\right) Y_{i j}^{\prime *} Y_{k l}^{\prime} V \xi_{k l}\right\rangle \\
= & \sum_{i j k l}\left\langle V \xi_{i j}, \pi\left(X_{i}^{*} X_{k}\right) T^{\prime}\left(Y_{i j}^{\prime *} Y_{k l}^{\prime}\right) V \xi_{k l}\right\rangle \\
\geq & \sum_{i j k l}\left\langle V \xi_{i j}, \pi\left(X_{i}^{*} X_{k}\right) T^{\prime}\left(Y_{i j}^{* *}\right) T^{\prime}\left(Y_{k l}^{\prime}\right) V \xi_{k l}\right\rangle
\end{aligned}
$$




$$
\begin{aligned}
& =\sum_{i j k l}\left\langle T^{\prime}\left(Y_{i j}^{\prime}\right) V \xi_{i j}, \pi\left(X_{i}^{*} X_{k}\right) T^{\prime}\left(Y_{k l}^{\prime}\right) V \xi_{k l}\right\rangle \\
& =\sum_{i j k l}\left\langle W^{\prime} Y_{i j}^{\prime} V \xi_{i j}, \pi\left(X_{i}^{*} X_{k}\right) W^{\prime} Y_{k l}^{\prime} V \xi_{k l}\right\rangle \\
& =\sum_{i j k l}\left\langle Y_{i j}^{\prime} V \xi_{i j}, W \pi\left(X_{i}^{*} X_{i}\right) W^{*} Y_{k l}^{\prime} V \xi_{k l}\right\rangle
\end{aligned}
$$

for all $X_{i} \in \mathcal{M}, Y_{i j}^{\prime} \in \pi(\mathcal{M})^{\prime}, \xi_{k l} \in \mathscr{K}$. Hence the map

$$
X \in \mathcal{M} \rightarrow \pi(T(X))-W \pi(X) W^{*}
$$

is completely positive.

Remark 4.13. Adopt the hypothesis of corollary 4.10 and let $S: \mathscr{A} \rightarrow \mathscr{A}$ be a completely positive map commuting with $\tau$ and which is the identity on $\mathscr{A}^{\tau}$. Then there exists a probability measure $\mu$ on $G$ such that

$$
S(X)=\int_{G} d \mu(g) \tau(g)(X), \quad \text { for all } X \text { in } \mathscr{A} .
$$

Let $U(g)$ be the canonical unitary representation of $G$ implementing $\tau$ in $\pi$, i.e.

$$
U(g) \pi(X) V=\pi(\tau(g)(X)) V,
$$

for all $X$ in $\mathscr{A}$. The action $\tau$ extends to a $\sigma$-weakly continuous action of $\mathcal{M}=\pi(\mathscr{A})^{\prime \prime}$, and

$$
P(X)=\int d g U(g) X U(g)^{*}
$$

is a $\sigma$-weakly continuous faithful projection from $\mathscr{M}$ onto $\mathcal{M}^{\tau}=\pi\left(\mathscr{A}^{\tau}\right)^{\prime \prime}$. The proof of corollary 4.10 shows that $S$ extends by normality to a completely positive normal map $T: M \rightarrow M$ such that

$$
T \operatorname{Ad}(U(g))=\operatorname{Ad}(U(g)) T, \quad T(X)=X
$$

for all $g$ in $G, X$ in $\mathscr{M}^{\tau}=P(\mathscr{M})$. This entails $P T=T$ and hence proposition 4.12 applies, to give

$$
X \rightarrow \pi(S(X))-W \pi(X) W^{*}
$$

is completely positive with

$$
\begin{aligned}
W \pi(X) V & =\pi\left(\int d \mu(g) \tau(g)(X)\right) V \\
& =\int d \mu(g) U(g) \pi(X) V
\end{aligned}
$$

i.e.

See also $[13, \S 5]$.

$$
W=\int_{G} d \mu(g) U(g) .
$$

\section{Dynamical semigroups}

In this section we analyse dynamical semigroups which commute with a compact action.

THEOREM 5.1. Let $G$ be a compact abelian group and $\tau$ an action of $G$ on a $C^{*}$-algebra $\mathscr{A}$, such that the ideals $\mathscr{A}^{\tau}(\gamma) \mathscr{A}^{\tau}(\gamma)^{*}$ in $\mathscr{A}^{\tau}$ are either dense or zero, for 
each $\gamma$ in $\hat{G}$, and such that the centre of $M\left(\mathscr{A}^{\tau}\right)$ is contained in the centre of $M(\mathscr{A})$. Let $H: \mathscr{A}_{F} \rightarrow \mathscr{A}$ be a ${ }^{*}$-linear map satisfying

(5.1) $\left[H\left(X_{i}^{*} X_{j}\right)\right] \leq\left[H\left(X_{i}\right)^{*} X_{j}+X_{i}^{*} H\left(X_{j}\right)\right]$

for all finite sequences $X_{1}, \ldots, X_{n}$ in $\mathscr{A}_{F}$.

(5.2) $H \tau(g)=\tau(g) H \quad$ for all $g$ in $G$.

(5.3) $H(X)=0$ for all $X$ in $\mathscr{A}^{\tau}$.

Then $H$ is closable, the closure $\bar{H}$ generates a $C_{0}$-semigroup of completely positive maps, and for each $t \geq 0$, there is a centre $\left(M\left(\mathscr{A}^{\tau}\right)\right)$-valued probability measure $\mu_{t}$ on $G$ such that

$$
\exp (-t \bar{H})(X)=\int_{G} d \mu_{t}(g) \tau(g)(X)
$$

for all $X$ in $\mathscr{A}$.

Remark 5.2. Conversely, if $H$ is the generator of a $C_{0}$-semigroup of completely positive maps which commute with $\tau$ and restrict to the identity map on $\mathscr{A}^{\tau}$, then $H$ trivially satisfies (5.2) and (5.3). But $\mathscr{A}_{F}$ is not necessarily contained in the domain $D(H)$ of $H$ ([6, example 6.2]). The generator $H$ will satisfy the inequality in (5.1) for all finite sequences $X_{1}, \ldots, X_{n}$ in $D(H)$ such that $X_{i}^{*} X_{i} \in D(H)$, for all $i, j=1, \ldots, n$, but the domain $D(H)$ is not a *algebra in general.

Proof. We follow closely the proof of theorem 4.2, and, as there, we may assume that $\tau$ is faithful, and $\mathscr{A}^{\tau}(\gamma) \mathscr{A}^{\tau}(\gamma)^{*}$ is dense in $\mathscr{A}^{\tau}$ for all $\gamma$ in $\hat{G}$.

Lemma 5.3. Adopt the hypothesis of theorem 5.1. Then

$$
H(X Y)=X H(Y), \quad H(Y X)=H(Y) X
$$

for all $X$ in $\mathscr{A}^{\tau}$ and $Y$ in $\mathscr{A}$.

Proof. Condition (5.1) implies that

$$
D(X, Y)=\phi\left(H(X)^{*} Y+X^{*} H(Y)-H\left(X^{*} Y\right)\right)
$$

is a non-negative sesquilinear form on $\mathscr{A}_{F}$ for each state $\phi$ on $\mathscr{A}_{F}$. If $X \in \mathscr{A}^{\tau}$, then $X^{*}, X X^{*} \in \mathscr{A}^{\tau}$ and (5.3) implies that

$$
D\left(X^{*}, X^{*}\right)=0 .
$$

As in lemma 4.3, we deduce

$$
H(X) Y+X H(Y)-H(X Y)=0,
$$

for all $X$ in $\mathscr{A}^{\tau}, Y$ in $\mathscr{A}_{F}$, and hence

$$
H(X Y)=X H(Y) .
$$

LEMMA 5.4. Adopt the hypothesis of theorem 5.1, and let $\mathscr{A}$ be faithfully and non-degenerately represented on a Hilbert space $\mathscr{H}$. Then there exists a closed, possibly unbounded operator $L(\gamma)$ on $\mathscr{H}$ such that

(5.5) $\mathscr{A}^{\tau}(\gamma) \mathscr{H} \subseteq D(L(\gamma))$, and $H(X)=L(\gamma) X, \quad$ for all $X$ in $\mathscr{A}^{\tau}(\gamma)$.

(5.6) $L(\gamma)$ is dissipative, i.e. $\operatorname{Re}\langle\xi, L(\gamma) \xi\rangle \geq 0, \quad$ for all $\xi \in D(L(\gamma))$.

(5.7) $L(\gamma)$ is affiliated with the abelian von Neumann algebra $\left(\mathscr{A}^{\top}\right)^{\prime \prime} \cap\left(\mathscr{A}^{\top}\right)^{\prime}$. 
Proof. As in the proof of lemma 4.4, let $E_{\alpha}$ be an approximate identity for $\mathscr{A}$ of the form

$$
E_{\alpha}=\sum_{i} A_{i}^{\alpha} A_{i}^{\alpha *}
$$

where $A_{i}^{\alpha} \in \mathscr{A}^{\tau}(\gamma)$, and each sum is finite. Define

$$
L_{\alpha}=\sum_{i} H\left(A_{i}^{\alpha}\right) A_{i}^{\alpha *} .
$$

If $X \in \mathscr{A}^{\top}(\gamma)$, then by lemma 5.3 :

$$
\begin{aligned}
L_{\alpha} X & =\sum_{i} H\left(A_{i}^{\alpha}\right) A_{i}^{\alpha *} X=\sum_{i} H\left(A_{i}^{\alpha} A_{i}^{\alpha *} X\right) \\
& =\sum_{i}^{\alpha} A_{i}^{\alpha} A_{i}^{\alpha *} H(X)=E_{\alpha} H(X),
\end{aligned}
$$

and so $\lim _{\alpha} L_{\alpha} X=H(X)$ for $X \in \mathscr{A}^{\tau}(\gamma)$. Define $L_{0}(\gamma)$ on $D\left(L_{0}(\gamma)\right)=\mathscr{A}^{\tau}(\gamma) \mathscr{H}$ by

$$
L_{0}(\gamma) \xi=\lim _{\alpha} L_{\alpha} \xi
$$

where the limit exists by the above reasoning. Then $L_{0}(\gamma)$ is densely defined because $\mathscr{A}^{\tau}(\gamma) \mathscr{A}^{\tau}(\gamma)^{*}$ contains an approximate identity for $\mathscr{A}$. If $X_{i} \in \mathscr{A}^{\tau}(\gamma), \eta_{i} \in \mathscr{H}$ and $\xi=\sum X_{i} \eta_{i}$, then

$$
\begin{aligned}
2 \operatorname{Re}\left\langle\xi, L_{0}(\gamma) \xi\right\rangle & =\left\langle\xi, L_{0}(\gamma) \xi\right\rangle+\left\langle L_{0}(\gamma) \xi, \xi\right\rangle \\
& =\sum_{i, j}\left\{\left\langle X_{i} \eta_{i}, L_{0}(\gamma) X_{j} \eta_{j}\right\rangle+\left\langle L_{0}(\gamma) X_{i} \eta_{i}, X_{j} \eta_{j}\right\rangle\right\} \\
& =\sum_{i, j}\left\langle\eta_{i},\left\{X_{i}^{*} H\left(X_{j}\right)+H\left(X_{i}\right)^{*} X_{i}\right\} \eta_{j}\right\rangle \\
& \geq \sum_{i, j}\left\langle\eta_{i}, H\left(X_{i}^{*} X_{i}\right) \eta_{j}\right\rangle=0
\end{aligned}
$$

where we have used (5.1) and (5.3). Thus $L_{0}(\gamma)$ is dissipative, hence closable, and its closure $L(\gamma)$ is dissipative [7, lemma 3.1.14].

Next we show that $L(\gamma)$ is affiliated with $\left(\mathscr{A}^{\top}\right)^{\prime \prime}$. Let

$$
\mathscr{D}=\mathscr{A}^{\alpha}(\gamma) \mathscr{A}^{\alpha}(\gamma)^{*} \mathscr{H} \subseteq D(L(\gamma)) .
$$

If $X, Y \in \mathscr{A}^{\tau}(\gamma), \eta \in \mathscr{H}$, then

$$
L(\gamma)\left(X Y^{*} \eta\right)=H(X) Y^{*} \eta .
$$

This last expression and the fact that $\mathscr{A}^{\tau}(\gamma)^{*} \mathscr{A}^{\tau}(\gamma)$ contains an approximate identity for $\mathscr{A}$ shows that $\mathscr{D}$ is a core for $L_{0}(\gamma)$, and so for $L(\gamma)$. As $\mathscr{A}^{\tau}(\gamma) \mathscr{A}^{\tau}(\gamma)^{*} \subseteq \mathscr{A}^{\tau}$, it follows that $\mathscr{D}$ is invariant under $\left(\mathscr{A}^{\top}\right)^{\prime}$. If $\xi \in \mathscr{D}$ and $X^{\prime} \in\left(\mathscr{A}^{\top}\right)^{\prime}$, then

$$
L_{\alpha} X^{\prime} \xi=X^{\prime} L_{\alpha} \xi
$$

as $L_{\alpha} \in \mathscr{A}^{\tau}$. Since $X^{\prime} \xi \in \mathscr{D}$, we may deduce

$$
L(\gamma) X^{\prime} \xi=X^{\prime} L(\gamma) \xi
$$

and so $L(\gamma) X^{\prime} \supseteq X^{\prime} L(\gamma)$ since $L(\gamma)$ is closed with core $\mathscr{D}$. Thus $L(\gamma)$ is affiliated with $\left(\mathscr{A}^{\top}\right)^{\prime \prime}$.

Finally we show that $L(\gamma)$ is affiliated with $\left(\mathscr{A}^{\tau}\right)^{\prime}$ (and hence with $\mathscr{A}^{\tau \prime \prime} \cap \mathscr{A}^{\tau \prime}$ ). If $X \in \mathscr{A}^{\tau}, Y \in \mathscr{A}^{\tau}(\gamma), \eta \in \mathscr{H}$, then $Y \eta$ and $X(Y \eta) \in D\left(L_{0}(\gamma)\right)$ and

$$
L_{0}(\gamma) X(Y \eta)=H(X Y) \eta=X H(Y) \eta=X L_{0}(\gamma)(Y \eta),
$$

and by taking closures, $L(\gamma) X \supseteq X L(\gamma)$, for all $X$ in $\mathscr{A}^{\tau}$. 
Remark 5.5. Multiplication on the left with $L(\gamma)$ determines a (possibly unbounded) multiplier on $\mathscr{A}^{\tau}$ and also on centre $\left(\mathscr{A}^{\tau}\right)$, (but this does not seem useful unless centre $\left(\mathscr{A}^{\tau}\right)$ contains an approximate identity of $\left.\mathscr{A}^{\tau}\right)$. To see this, note that the ideal $\mathscr{A}^{\tau}(\gamma) \mathscr{A}^{\tau}(\gamma)^{*}$ must contain the minimal dense ideal $K\left(\mathscr{A}^{\tau}\right)$ of $\mathscr{A}^{\tau},[26,5.6]$. If

$$
f:[0, \infty) \rightarrow[0, \infty)
$$

is continuous with $f(x)=0$ for small $x$, and $X=X^{*} \in \mathscr{A}^{\tau}$ then

$$
f(X) \mathscr{A}^{\tau}(\gamma) \mathscr{A}^{\tau}(\gamma)^{*} f(X) \in K\left(\mathscr{A}^{\tau}\right)
$$

and as $L(\gamma)$ commutes with $f(X)$ it follows that $K\left(\mathscr{A}^{\tau}\right)$ is a core for the operation of left multiplication by $L(\gamma)$, on $\mathscr{A}^{\tau}(\gamma) \mathscr{A}^{\tau}(\gamma)^{*}$. As $L(\gamma)$ commutes with all elements in $\mathscr{A}^{\tau}$ it follows that

$$
X L(\gamma) Y=L(\gamma) X Y \quad \text { for all } X \text { and } Y \in K\left(\mathscr{A}^{\tau}\right) .
$$

As

$$
L(\gamma) f(X) \mathscr{A}^{\tau}(\gamma) \mathscr{A}^{\top}(\gamma)^{*} f(X)=f(X) L(\gamma) \mathscr{A}^{\tau}(\gamma) \mathscr{A}^{\tau}(\gamma)^{*} f(X)
$$

when $f, X$ are as above, it follows easily that

$$
L(\gamma) K\left(\mathscr{A}^{\tau}\right) \subseteq K\left(\mathscr{A}^{\tau}\right) .
$$

By $[4,2.5], L(\gamma)$ is an unbounded multiplier of $\mathscr{A}^{\top}$. Now it follows immediately from $[26,5.6 .1]$ that

$$
K(\text { centre }(\mathscr{A})) \subseteq \operatorname{centre}\left(K\left(\mathscr{A}^{\tau}\right)\right)
$$

Then as centre $K\left(\mathscr{A}^{\tau}\right)$ is contained in the domain of the multiplier defined by $L(\gamma)$, and $L(\gamma)$ commutes with $\mathscr{A}^{\tau}$, it follows that

$$
L(\gamma) K\left(\text { centre }\left(\mathscr{A}^{\tau}\right)\right) \subseteq K\left(\text { centre }\left(\mathscr{A}^{\tau}\right)\right)
$$

and hence $L(\gamma)$ defines an unbounded multiplier on $K$ (centre $(\mathscr{A}))$.

Lemma 5.6. Adopt the hypothesis of theorem 5.1. In any faithful non-degenerate representation of $\mathscr{A}$ on a Hilbert space $\mathscr{H}$, the operator $L(\gamma)$ of lemma 5.4 has the properties:

(5.8a) $L(\gamma)$ is the generator of a $C_{0}$-semigroup $\exp (-t L(\gamma))$ on $\mathscr{H}$.

(5.8b) $\exp (-t L(\gamma))$ is contained in centre $M\left(\mathscr{A}^{\tau}\right)$ for all $t \geq 0$.

Proof. By lemma 5.4, $L(\gamma)$ is dissipative, and $L(\gamma)$ is affiliated with the abelian von Neumann algebra $\mathscr{A}^{\top} \cap \cap \mathscr{A}^{\tau \prime}$, and so $L(\gamma)$ is the generator of a contraction semigroup in $\mathscr{A}^{\tau \prime \prime} \cap \mathscr{A}^{\tau \prime},\left[\right.$ [30], [31]. To show that $\exp (-t L(\gamma)) \mathscr{A} \subseteq \mathscr{A}$, and $\exp (-\mathrm{tL}(\gamma)) \mathscr{A}^{\tau} \subseteq \mathscr{A}^{\tau}$, we will produce a dense set of analytic elements for the unbounded multiplier defined by $L(\gamma)$ on $\mathscr{A}$. Let $A_{1}, \ldots, A_{n} \in \mathscr{A}^{\tau}(\gamma)$, and $f:[0, \infty) \rightarrow[0, \infty)$ a bounded continuous function such that for some $\varepsilon>0, f(x)=0$ if $x<\varepsilon$. We show that

$$
A=f\left(\sum_{i=1}^{n} A_{i} A_{i}^{*}\right) \sum_{i=1}^{n} A_{i} A_{i}^{*} \in \mathscr{A}^{\tau}
$$

is analytic for the left multiplier defined by $L(\gamma)$. Let $E_{\varepsilon}$ be the spectral projection of $\sum_{i=1}^{n} A_{i} A_{i}^{*}$ corresponding to $[\varepsilon, \infty)$. As

$$
L(\gamma)\left(\sum_{i=1}^{n} A_{i} A_{i}^{*}\right)=\sum_{i=1}^{n} H\left(A_{i}\right) A_{i}^{*}
$$


is a bounded operator and

$$
\sum_{i=1}^{n} A_{i} A_{i}^{*} \geq \varepsilon E_{\varepsilon}
$$

it follows that $L(\gamma) E_{\varepsilon}$ is bounded, i.e.

$$
\left\|L(\gamma) E_{\varepsilon}\right\|=K<\infty
$$

Now $E_{\varepsilon} \in \mathscr{A}^{\tau \prime \prime}$, and so $L(\gamma)$ and $E_{\varepsilon}$ commute and hence

$$
\left\|L(\gamma)^{m} E_{\varepsilon}\right\| \leq K^{m}, \quad \text { for all } m \text {. }
$$

But

$$
f\left(\sum_{i=1}^{n} A_{i} A_{i}^{*}\right) \leq\|f\|_{\infty} E_{\varepsilon}
$$

and so

$$
\begin{aligned}
\left\|L(\gamma)^{m} A\right\| & \leq\left\|L(\gamma)^{m} f\left(\sum_{i=1}^{n} A_{i} A_{i}^{*}\right)\right\|\left\|\sum_{i=1}^{n} A_{i} A_{i}^{*}\right\| \\
& \leq\|f\|_{\infty} K^{m}\left\|\sum_{i=1}^{n} A_{i} A_{i}^{*}\right\| .
\end{aligned}
$$

Thus $A$ is entire analytic for the multiplier defined by $L(\gamma)$.

Now $\mathscr{A}^{\tau}$, (and hence $\mathscr{A}$ ), has an approximate identity consisting of elements of the form

$$
E_{\alpha}=f_{\alpha}\left(\sum_{i} A_{i}^{\alpha} A_{i}^{\alpha *}\right)\left(\sum_{i} A_{i}^{\alpha} A_{i}^{\alpha *}\right)
$$

where $A_{i}^{\alpha} \in \mathscr{A}^{\top}(\gamma)$, and $f_{\alpha}$ is a continuous positive function which is zero on an interval $\left[0, \varepsilon_{\alpha}\right)$, one on an interval $\left[2 \varepsilon_{\alpha}, \infty\right)$, for some $\varepsilon_{\alpha}>0$. Each $E_{\alpha}$ is then entire analytic for both the left and right multiplier defined by $L(\gamma)$. Thus if $X \in \mathscr{A}^{\tau}$, one has:

$$
\exp (-t L(\gamma)) E_{\alpha} X=\left(\sum_{n=0}^{\infty} \frac{(-t L(\gamma))^{n}}{n !} E_{\alpha}\right) X \quad \in \mathscr{A}^{\tau}
$$

and as $\exp (-t L(\gamma))$ is bounded and $E_{\alpha}$ is an approximate identity, one deduces

$$
\exp (-t L(\gamma)) X \in \mathscr{A}^{\tau}
$$

Similarly

$$
X \exp (-t L(\gamma))=\lim _{\alpha} X E_{\alpha} \exp (-t L(\gamma)) \quad \in \mathscr{A}^{\tau}
$$

i.e. $\exp (-t L(\gamma))$ is a multiplier of $\mathscr{A}^{\tau}$. Finally as $L(\gamma)$ is affiliated with $\mathscr{A}^{\tau \prime}$, we have exp $(-t L(\gamma)) \in \mathscr{A}^{\tau}$ and as $\mathscr{A}^{\tau}$ is dense in $M\left(\mathscr{A}^{\tau}\right)$ in the strict topology, it follows that

$$
\exp (-t L(\gamma)) \in \operatorname{centre}\left(M\left(\mathscr{A}^{\tau}\right)\right)
$$

LemMa 5.7. Adopt the hypothesis of theorem 5.1. For each $t \geq 0$, the function

$$
\gamma \in \hat{G} \rightarrow \exp (-t L(\gamma)) \in \operatorname{centre}\left(M\left(\mathscr{A}^{\tau}\right)\right)
$$

is positive definite. 
Proof. As all the $L(\gamma)$ 's are affiliated with $\mathscr{A}^{\tau \prime \prime} \cap \mathscr{A}^{\tau \prime}$, the $L(\gamma)$ can be thought of as functions in a common spectral representation. We first show that $L$ is negative definite in the sense that the $n \times n$ matrix

$$
\left[L\left(\gamma_{j}-\gamma_{i}\right)-L\left(\gamma_{j}\right)-L\left(\gamma_{i}\right)^{*}\right]
$$

is negative for all $\gamma_{i} \cdots \gamma_{n} \in \hat{G}$, and all $n \geq 1$, [5, definition 7.1].

If $X_{i} \in \mathscr{A}^{\tau}\left(\gamma_{i}\right)$ for $i=1, \ldots, n$, then by (5.1)

$$
\left[H\left(X_{i}^{*} X_{i}\right)-H\left(X_{i}\right)^{*} X_{j}-X_{i}^{*} H\left(X_{j}\right)\right] \leq 0
$$

i.e.

$$
\left[L\left(\gamma_{j}-\gamma_{i}\right) X_{i}^{*} X_{i}-X_{i}^{*} L\left(\gamma_{i}\right)^{*} X_{j}-X_{i}^{*} L\left(\gamma_{j}\right) X_{j}\right] \leq 0 .
$$

By lemma 5.6, $\exp (-t L(\gamma)) \in$ centre $M\left(\mathscr{A}^{\tau}\right)$, and so $\exp (-t L(\gamma)) \in$ centre $M(\mathscr{A})$ by the hypotheses of theorem 5.1. Thus $\exp (-t L(\gamma))$ commutes with $X_{i}^{*}$ for $i=$ $1, \ldots, n$, and so $L(\gamma)$ commutes strongly with $X_{i}^{*}$ for all $\gamma$, i.e.

$$
L\left(\gamma_{j}-\gamma_{i}\right) X_{i}^{*} \supseteq X_{i}^{*} L\left(\gamma_{j}-\gamma_{i}\right) .
$$

Thus

$$
\left[X_{i}^{*} L\left(\gamma_{j}-\gamma_{i}\right) X_{i}-X_{i}^{*} L\left(\gamma_{i}\right)^{*} X_{j}-X_{i}^{*} L\left(\gamma_{j}\right) X_{j}\right] \leq 0 .
$$

Using the same matrix tricks as in the proof of lemma 4.5 , we now deduce

$$
\left[\left(\sum_{k} X_{i}^{k} X_{i}^{k *}\right)\left(L\left(\gamma_{j}-\gamma_{i}\right)-L\left(\gamma_{i}\right)^{*}-L\left(\gamma_{j}\right)\right)\left(\sum_{k} X_{i}^{k} X_{j}^{k *}\right)\right] \leq 0
$$

for all finite sequences $X_{i}^{k} \in \mathscr{A}^{\tau}\left(\gamma_{i}\right)$, and the matrix operator has finite norm if

$$
\sum_{k} X_{i}^{k} X_{i}^{k *} \in K\left(\mathscr{A}^{\tau}\right) \quad \text { for all } i .
$$

Letting $\sum_{k} X_{i}^{k} X_{i}^{k *}$ run through an approximate identity we deduce

$$
\left[L\left(\gamma_{j}-\gamma_{i}\right)-L\left(\gamma_{i}\right)^{*}-L\left(\gamma_{j}\right)\right] \leq 0,
$$

i.e. $L$ is negative definite. As $L(0)=0$, it follows from Schoenberg's Theorem that $\exp (-t L(\cdot))$ is positive definite for all $t \geq 0,[5$, theorem 7.8].

End of proof of theorem 5.1. By lemmas 5.7 and 3.1, there exists for each $t \geq 0$, a centre $\left(M\left(\mathscr{A}^{\top}\right)\right)$-valued probability measure $\mu_{t}$ on $G$ such that

$$
\exp (-t L(\gamma))=\mu_{t}(\langle\gamma, \cdot\rangle)=\int_{G} d \mu_{t}(g)\langle\gamma, g\rangle .
$$

By $\S 3$, there exists a family $\left\{S_{t}: t \geq 0\right\}$ of completely positive maps on $\mathscr{A}$ defined by

$$
S_{t}(X)=\int d \mu_{t}(g) \tau(g)(X), \quad X \in \mathscr{A} .
$$

For $X \in \mathscr{A}^{\tau}(\gamma)$, we have

$$
S_{\mathrm{t}}(X)=\int d \mu_{t}(g)\langle\gamma, g\rangle X=\exp (-t L(\gamma))(X) .
$$

Thus $t \rightarrow S_{t}$ is a semigroup on $\mathscr{A}_{F}$, and since each $S_{t}$ is a contraction it follows by continuity that $t \rightarrow S_{t}$ is a semigroup on $\mathscr{A}$. That $S$ is strongly continuous follows from eq. (A). 
Furthermore, if $X \in \mathscr{A}^{\tau}(\gamma), \xi \in \mathscr{H}$, then $X \xi \in D(L(\gamma))$ and hence

$$
\begin{aligned}
S_{t}(X) \xi-X \xi & =\exp (-t L(\gamma)) X \xi-X \xi \\
& =-\int_{0}^{t} d s \exp (-s L(\gamma)) L(\gamma) X \xi .
\end{aligned}
$$

But $L(\gamma) X=H(X) \in \mathscr{A}^{\tau}(\gamma)$, and $s \rightarrow \exp (-s L(\gamma)) H(X)$ is continuous. Hence $X$ lies in the domain $D(\tilde{H})$ of the generator of $S$, and

$$
\tilde{H}(X)=\lim _{t \rightarrow 0} \frac{1}{t}\left(S_{t}(X)-X\right)=H(X) .
$$

Thus $H \subseteq \tilde{H}$, and $H$ is closable. Since $\mathscr{A}_{F}$ is invariant under $S$, it forms a core for $\tilde{H}$ [7, corollary 3.17], and so $\tilde{H}$ is the closure of $H$. This completes the proof of theorem 5.1.

COROllary 5.8. Let $G$ be a compact abelian group and $\tau$ a faithful action of $G$ on a $C^{*}$-algebra $\mathscr{A}$. Assume either

(5.9) $\mathscr{A}^{\top}$ is simple

or

(5.10) There exists a faithful representation $\pi_{0}$ of $\mathscr{A}^{\tau}$ such that $\pi\left(\mathscr{A}^{\tau}\right)^{\prime \prime}$ is a factor, if $\pi$ is the Stinespring representation of $\pi_{0} \circ P$.

Let $H: D(H) \subseteq \mathscr{A} \rightarrow \mathscr{A}$ be a densely defined closed *-linear map. Then the following four conditions (5.11)-(5.14) are equivalent:

(5.11) (a) $\mathscr{A}_{F} \subseteq D(H), \mathscr{A}_{F}$ is a core for $H$, and

$$
\left[H\left(X_{i}^{*} X_{j}\right)\right] \leq\left[H\left(X_{i}\right)^{*} X_{j}+X_{i}^{*}\left(X_{j}\right)\right]
$$

for all finite sequences $X_{1}, \ldots, X_{n}$ in $\mathscr{A}_{F}$;

(b) $H \tau(g)=\tau(g) H \quad$ for all $g$ in $G$;

(c) $H(X)=0$ for all $X$ in $\mathscr{A}^{\tau}$.

(5.12) $\mathscr{A}_{F} \subseteq D(H), \mathscr{A}_{F}$ is a core for $H$, and there exists a negative definite function $\lambda$ from $\hat{G}$ into $\mathbb{C}$ such that $\lambda(0)=0$ and $H(X)=\lambda(\gamma) X$ for all $X$ in $\mathscr{A}^{\tau}(\gamma), \gamma$ in $\hat{G}$.

(5.13) $H$ is the generator of a strongly continuous semigroup on $\mathscr{A}$ such that

(a) $\exp (-t H)$ is completely positive for each $t \geq 0$;

(b) $\tau(g) \exp (-t H)=\exp (-t H) \tau(g), \quad$ for all $t \geq 0, g$ in $G$;

(c) $\exp (-t H)(X)=X, \quad$ for all $t \geq 0, X$ in $\mathscr{A}^{\tau}$.

(5.14) There exists a convolution semigroup $\left\{\mu_{t}: t \geq 0\right\}$ of probability measures on $G$ such that $H$ is the generator of the strongly continuous semigroup $S$ given by

$$
S_{t}(X)=\int_{G} d \mu_{t}(g) \tau(g)(X)
$$

for $t \geq 0, X$ in $\mathscr{A}$.

Furthermore, if $G$ is the d-dimensional torus $\mathbb{T}^{d}$ and $\left\{\partial / \partial t_{i}\right\}_{i=1}^{d}$ are the generators of the actions of the canonical one-parameter subgroups of $\mathrm{J}^{d}$ on $\mathscr{A}$, then conditions (5.11)-(5.14) are also equivalent to:

(5.15) $\mathscr{A}_{F} \subseteq D(H), \mathscr{A}_{F}$ is a core for $H$, and there is a triple $(b, a, \mu)$ where $b=\left(b_{1}, \ldots, b_{d}\right)$ is a d-tuple of real numbers, $a=\left[a_{i j}\right]$ is a real positive $d \times d$ matrix, 
and $\mu$ is a non-negative bounded measure on

$$
\mathbb{T}^{d} \backslash\{0\}=\left\{x=\left(x_{i}\right):-\pi<x_{i} \leq \pi, x \neq 0\right\}
$$

such that

$$
H(X)=\left\{\sum_{k=1}^{d} b_{k} \frac{\partial}{\partial t_{k}}-\sum_{i j} a_{i j} \frac{\partial^{2}}{\partial t_{i} \partial t_{j}}+\int_{\mathbb{T}^{d} \backslash\{0\}} \frac{d \mu(x)}{\|x\|^{2}}\left[1+\sum_{k} x_{k} \frac{\partial}{\partial t_{k}}-\exp \left(\sum_{k} x_{k} \frac{\partial}{\partial t_{k}}\right)\right]\right\} X
$$

where $\|x\|^{2}=\sum_{i=1}^{d} x_{i}^{2}$.

The triple in condition (5.15) is arbitrary within the constraints given there and is uniquely determined by $H$.

Proof. Assume first that $\mathscr{A}^{\tau}$ is simple so that as in corollary $4.6, \mathscr{A}^{\tau}(\gamma) \mathscr{A}^{\tau}(\gamma)^{*}$ is dense for each $\gamma \in \hat{G}$, and the centre of $M\left(\mathscr{A}^{\tau}\right)$ is trivial. Thus theorem 5.1 applies, the function $Z$ of lemmas 4.4 and 4.5 must be scalar-valued as well as the measures $\mu_{t .}$. This establishes $(5.11) \Leftrightarrow(5.12) \Rightarrow(5.14)$. The equivalence of (5.14) with (5.13) is a consequence of corollary 4.6 . To show $(5.14) \Rightarrow(5.12)$, suppose the convolution semigroup $\mu_{t}$ is given, so that by [5, theorem 8.3$]$ there is an unique negative definite function $\lambda$ on $\hat{G}$ such that

$$
\exp (-t \lambda(\gamma))=\int d \mu_{t}(g)\langle\gamma, g\rangle
$$

Then

$$
S_{t}(X)=\int d \mu_{t}(g)\langle\gamma, g\rangle X=\exp (-t \lambda(\gamma)) X \quad \text { for } X \in \mathscr{A}^{\tau}(\gamma)
$$

If $H$ is the generator of $S$ it follows that $X \in D(H)$, and $H(X)=\lambda(\gamma) X$. Hence $\mathscr{A}_{F} \subseteq D(H)$ and as $\mathscr{A}_{F}$ is $S$-invariant, it is a core for $H$, and so (5.12) holds.

To prove $(5.12) \Leftrightarrow(5.15)$, we use the Lévy-Khinchin representation of a negative definite function [5], [25]. If $G$ is a compact abelian group, and $\lambda$ is a negative definite function from $\hat{G}$ into $\mathbb{C}$, then $\lambda$ has a unique representation

$$
\lambda(\gamma)=c+\mathrm{i} l(\gamma)+q(\gamma)+\int_{G \backslash\{0\}} d \mu(g)(1+\mathrm{i} \psi(\gamma, g)-\langle\gamma, g\rangle)
$$

where

(5.16) $c \geq 0$ is a constant.

(5.17) $l$ is an additive linear functional from $\hat{G}$ into $\mathbb{R}$.

(5.18) $q$ is a non-negative quadratic form on $\hat{G}$, i.e.

$$
\begin{aligned}
& q\left(\gamma_{1}+\gamma_{2}\right)+q\left(\gamma_{1}-\gamma_{2}\right)=2\left[q\left(\gamma_{1}\right)+q\left(\gamma_{2}\right)\right], \quad \gamma_{1}, \gamma_{2} \in \hat{G}, \\
& q(\gamma) \geq 0, \quad \gamma \in \hat{G} .
\end{aligned}
$$

(5.19) $\psi$ is a fixed (independent of $\mu$ ) function on $\hat{G} \times G$ with the properties: $g \rightarrow \psi(\gamma, g)$ is continuous, $\gamma \rightarrow \psi(\gamma, g)$ is an additive character on $\hat{G}$ for each fixed $g \in G$, and

$$
\psi(\gamma,-g)=-\psi(\gamma, g)
$$

If $C$ is a finite subset of $\hat{G}$, there is a neighbourhood $N_{c}$ around 0 in $G$ such that

$$
\langle\gamma, g\rangle=\exp (\mathrm{i} \psi(\gamma, g))
$$

for all $\gamma \in C, g \in N_{c}$. 
(5.20) $\mu$ is a positive measure on $G \backslash\{0\}$ such that for all $\gamma$ in $\hat{G}$,

$$
\int_{G} d \mu(g)[1-\operatorname{Re}\langle\gamma, g\rangle]<\infty
$$

and $\mu$ is finite outside every neighbourhood of 0 .

In the case $G=\mathbb{V}^{d}$, one has $\hat{G}=\mathbb{Z}^{d}$, and if $G$ is identified with $\mathbb{R}^{d} /(2 \pi \mathbb{Z})^{d}$ one has

$$
\langle\gamma, g\rangle=\left\langle n_{1}, \ldots, n_{d} ; x_{1}, \ldots, x_{d}\right\rangle=\exp \mathrm{i}\left(\sum_{k=1}^{d} n_{k} x_{k}\right),
$$

when $-\pi<x_{k} \leq \pi$. ( $\psi$ is then not continuous nor antisymmetric in $g$ but these properties are not essential for the Lévy-Khinchin representation, only the asymptotic properties of $\psi$ for $g$ near 0 , and the fact that $\psi(\cdot, g)$ is an additive character are essential.) Thus an arbitrary negative definitive function on $\hat{\mathbb{V}}^{d}=\mathbb{Z}^{d}$ has the form

$$
\begin{aligned}
\lambda(\gamma)= & \lambda\left(n_{1}, \ldots, n_{d}\right) \\
= & c+\mathrm{i} \sum_{k=1}^{d} b_{k} n_{k}+\sum_{i, j=1}^{d} a_{i j} n_{i} n_{j} \\
& +\int_{\tau^{d} \backslash\{0\}} d \mu(x)\left[1+\mathrm{i} \sum_{k=1}^{d} n_{k} x_{k}-\exp \left\{\mathrm{i} \sum_{i=1}^{d} n_{k} x_{k}\right\}\right],
\end{aligned}
$$

where $c \geq 0, b=\left(b_{1}, \ldots, b_{d}\right) \in \mathbb{P}^{d}, a=\left[a_{i j}\right]$ is a positive matrix in $M_{d}(\mathbb{R})$, and $\mu$ is a positive measure on $G \backslash\{0\}$ such that $\mu$ is finite outside every neighbourhood of 0 , and

$$
\left.\infty>\int_{G} d \mu(x)[1-\operatorname{Re}(\gamma, g)]=\int_{G} d \mu(x)\left[\frac{1}{2}\left(\sum n_{i} x_{i}\right)\right)^{2}+\text { higher order terms }\right] .
$$

Thus replacing $d \mu(x)$ by $d \mu(x) /\|x\|^{2}$ we may write

$$
\begin{aligned}
\lambda(\gamma)=c & +\mathrm{i} \sum_{k=1}^{d} b_{k} n_{k}+\sum_{i, j=1}^{d} a_{i j} n_{i} n_{j} \\
& +\int_{T^{d} \backslash\{0\}} \frac{d \mu(x)}{\|x\|^{2}}\left[1+\mathrm{i} \sum_{k=1}^{d} n_{k} x_{k}-\exp \left\{\mathrm{i} \sum_{k=1}^{d} n_{k} x_{k}\right\}\right]
\end{aligned}
$$

where the condition on $\mu$ is now that it is a finite positive measure.

If $X \in \mathscr{A}^{\tau}(\gamma)=\mathscr{A}^{\tau}\left(n_{1}, \ldots, n_{d}\right)$ we have:

$$
\frac{\partial}{\partial t_{k}} \tau(g) X=i n_{k} \tau(g) X
$$

Thus

$$
\begin{aligned}
H \tau(g)(X)= & \lambda(\gamma) \tau(g) X \\
= & \left\{c+\sum_{k=1}^{d} b_{k} \frac{\partial}{\partial t_{k}}-\sum_{i, j=1}^{d} a_{i j} \frac{\partial^{2}}{\partial t_{i} \partial t_{j}}\right. \\
& \left.\quad+\int_{\nabla^{d} \backslash\{0\}} \frac{d \mu(x)}{\|x\|^{2}}\left[1+\sum_{k=1}^{d} x_{k} \frac{\partial}{\partial t_{k}}-\exp \left(\sum_{k=1}^{d} x_{k} \frac{\partial}{\partial t_{k}}\right)\right]\right\} \tau(g)(X) .
\end{aligned}
$$

Finally, the condition $H \mid \mathscr{A}^{\tau}=0$ means $\lambda(0)=0$, and this is equivalent to $c=0$ in the Lévy-Khinchin representation for $\lambda$. This establishes the equivalence of $(5.12)$ with (5.15). 
In the case that there exists a faithful representation $\pi_{0}$ of $\mathscr{A}^{\tau}$ such that $\pi\left(\mathscr{A}^{\tau}\right)^{\prime \prime}$ is a factor, where $\pi$ is the Stinespring representation associated to $\pi_{0}{ }^{\circ} P$, corollary 5.8 is proved from a von Neumann version of theorem 5.1, using the same techniques as in proving corollary 4.10 from corollary 4.8 . This von Neumann version is:

Remark 5.9. Let $G$ be a compact abelian group, and $\tau$ an action of $G$ on a von Neumann algebra $\mathscr{M}$ such that $\mathcal{M}^{\tau}$ is a factor. Let

$$
H: D(H) \subseteq \mathscr{M} \rightarrow \mathscr{M}
$$

be a $\sigma$-weakly densely defined, $\sigma$-weakly- $\sigma$-weakly closed operator. Then the conditions (5.11)-(5.15) of corollary 5.8, with the obvious modifications, are again equivalent.

The modifications in the proof are the same as in the proof of corollary 4.8 .

By using the concepts introduced prior to theorem 5.1 in [9] and the general Lévy-Khinchin formula one can formulate an analogue of condition 5 for a general compact abelian group $G$. We omit the details.

REMARK 5.10. Let $\mathscr{A}$ be a $C^{*}$-algebra on a Hilbert space $\mathscr{H}$. It is known [11] that if $H$ is the bounded generator of a norm continuous semigroup of completely positive maps on $\mathscr{A}$, then

$$
H=-K+L(\cdot)+(\cdot) L^{*},
$$

where $K$ is completely positive from $\mathscr{A}$ into $\mathscr{A}^{\prime \prime}$, and $L \in \mathscr{A}^{\prime \prime}$. Equivalently, there exists $L \in \mathscr{A}^{\prime \prime}$, such that

$$
X \rightarrow \exp (-t H)(X)-\exp (-t L)(X) \exp \left(-t L^{*}\right)
$$

is completely positive from $\mathscr{A}$ into $\mathscr{A}^{\prime \prime}$, for all $t \geq 0$.

Now suppose $\tau$ is an action of a compact (abelian) group $G$ on a $C^{*}$-algebra $\mathscr{A}$, and let $S_{t}$ be a $C_{0}$-semigroup on $\mathscr{A}$ such that

(5.21) $S_{t}$ is completely positive for each $t \geq 0$;

(5.22) $S_{t} \tau(g)=\tau(g) S_{t} \quad$ for all $g$ in $G, t \geq 0$;

(5.23) $S_{t}(X)=X \quad$ for all $X$ in $\mathscr{A}^{\tau}, t \geq 0$.

Let $\pi_{1}$ be a faithful representation of $\mathscr{X}^{\top}$ on a Hilbert space $\mathscr{K}$, and $(\pi, \mathscr{H}, V)$ the Stinespring decomposition of $\pi_{0}{ }^{\circ} P$, where $P: \mathscr{A} \rightarrow \mathscr{A}^{\tau}$ is the canonical projection. By the proof of proposition 4.12 , there is a contraction $W_{t}$ on $\mathscr{H}$ given by

$$
W_{t} \pi(X) V=\pi\left(S_{\mathrm{t}}(X)\right) V, \quad X \in \mathscr{A}, t \geq 0,
$$

and there is a unitary representation $U$ of $G$ on $\mathscr{H}$ given by

$$
U(g) \pi(X) V=\pi(\tau(g)(X)) V
$$

such that $(\pi, U)$ is $\tau$-covariant. Then $\tau$ extends to an action $\hat{\tau}$ of $G$ on $\mathscr{M}=\pi(\mathscr{A})^{\prime \prime}$, and the projection $P$ extends to $\mathscr{M}$ through the formula

$$
\hat{P}=\int d g \hat{\tau}(g) \text {. }
$$

This formula shows that $\hat{P}$ is a faithful positive map with range $\mathscr{M}^{\tau}=\pi\left(\mathscr{A}^{\top}\right)^{\prime \prime}$. The representation $\pi_{0}$ of $\mathscr{A}^{\tau}$ is unitarily equivalent to the restriction of $\pi$ to $\mathscr{A}^{\tau}$ on $V \mathscr{K}$, using the isometry $V$ between $\mathscr{K}$ and $V \mathscr{K}$. It follows that the identity representation 
of $\mathscr{M}$ on $\mathscr{H}$ identifies with the Stinespring representation associated to $\hat{\pi}_{0} \circ \hat{P}$, where $\hat{\pi}_{0}$ is the extension of $\pi_{0}$ to $\mathscr{M}^{\tau}$, defined by the above unitary equivalence. But $\hat{P}$ is faithful and so $V \mathscr{K}$ is separating for $\mathcal{M}$, and hence cyclic for $\mathcal{M}^{\prime}$. Then

$$
\pi S_{t}(X) V=W_{t} \pi(X) V
$$

implies that $S_{t}^{*}$ maps the normal states in the representation $\pi$ into normal states, and so $S_{t}$ extends by $\sigma$-weak continuity to $\hat{S}_{t}$ on $\mathcal{M}$, such that

(5.24) $\hat{S}_{t}$ is completely positive for each $t \geq 0$;

(5.25) $\hat{\tau}(g) \hat{S}_{t}=\hat{S} \hat{\tau}(g) \quad$ for all $g$ in $G, t \geq 0$;

(5.26) $\hat{P} \hat{S}_{t}=\hat{P} \quad$ for all $t \geq 0$.

Moreover

$$
W_{t} X V=\hat{S}_{t}(X) V, \quad X \in \mathcal{M}, t \geq 0,
$$

and by proposition 4.12 it follows that

$$
X \in \mathcal{M} \rightarrow \hat{S}_{t}(X)-W_{t} X W_{t}^{*}
$$

is completely positive. In particular the map

$$
X \in \mathscr{A} \rightarrow \pi\left(S_{t}(X)\right)-W_{t} \pi(X) W_{t}^{*}
$$

is completely positive. Let $L$ denote the generator of the $C_{0}$-semigroup, $W_{t}$, so that $L^{*}$ is the generator of the $C_{0}$-semigroup $W_{t}^{*}$. If $x \in D(H)$, we may define a bilinear form $K(X)$ on $D\left(L^{*}\right) \times D\left(L^{*}\right)$ by

$$
\begin{aligned}
\langle\xi, K(X) \eta\rangle & =\lim _{t \rightarrow 0} \frac{1}{t}\left\langle\xi,\left\{\pi\left(S_{t}(X)\right)-W_{t} \pi(X) W_{t}^{*}\right\} \eta\right\rangle \\
& =-\langle\xi, \pi(H(X)) \eta\rangle+\left\langle L^{*} \xi, \pi(X) \eta\right\rangle+\left\langle\xi, \pi(X) L^{*} \eta\right\rangle .
\end{aligned}
$$

So, suppressing the symbol $\pi$,

$$
H(X)=-K(X)+L X+X L^{*}
$$

as bilinear forms, and $K$ is a completely positive map from $D(H)$ into unbounded bilinear forms in an obvious manner.

In many cases, the above form identity makes sense as an operator identity on a dense subspace of $\mathscr{H}$. Assume for example that $\mathscr{A}$ is unital,

$$
\mathscr{A}^{\tau}(\gamma) \mathscr{A}^{\tau}(\gamma)^{*}=\mathscr{A}^{\tau}
$$

for each $\gamma$ in $\hat{G}$, and $\mathscr{A}_{F} \subseteq D(H)$. Then since $\mathscr{A}^{\tau}$ is unital, the multipliers $L(\gamma)$ in the proof of theorem 5.1 must be bounded. If $X \in \mathscr{A}^{\tau}(\gamma)$, then

$$
\exp (t L) \pi(X) V=\pi \exp (t L(\gamma)) X) V
$$

and so

and

$$
\mathscr{H}_{0}=\pi\left(\mathscr{A}^{\tau}(\gamma)\right) V \mathscr{H} \subseteq D(L),
$$

$$
L \pi(X) V=\pi(L(\gamma) X) V .
$$

Hence $L, L^{*}$ map $\mathscr{H}_{0}$ into itself and

$$
H(X)=-K(X)+L X+X L^{*}
$$

makes sense as an operator identity on $\mathscr{H}_{0}$ when $X \in \mathscr{A}_{F}$.

That $K(X)$ really can be unbounded is illustrated by the following example: let $G=\mathbb{T}, \mathscr{A}=C(\mathbb{T}), \tau$ be translation, and $H=-d^{2} / d x^{2}$ the generator of the diffusion 
semigroup. Then $\mathscr{A}^{\tau}=\mathbb{C}$, and $\mathscr{A}$ has a unique $G$-invariant state given by Haar measure on $\pi$. If $\pi_{0}$ is the one-dimensional representation of $\mathscr{A}^{\tau}$, then $\mathscr{H}=L^{2}(\mathbb{T})$, and if $\psi \in L^{2}$,

$$
L \psi=-\psi^{\prime \prime}=L^{*} \psi
$$

If $f \in C^{2}(\mathbb{T}), f \in D(H)$ and $H f=-f^{\prime \prime}$. If $\psi \in C^{2}(\mathbb{T})$ then $\psi \in D\left(L^{*}\right), f \psi \in D(L)$ and

$$
\left(L f+f L^{*}\right) \psi=-f^{\prime \prime} \psi-2 f^{\prime} \psi^{\prime}-2 f \psi^{\prime \prime} .
$$

Thus

$$
K(f) \psi=-2 f^{\prime} \psi^{\prime}-2 f \psi^{\prime \prime},
$$

which shows that $K(f)$ is unbounded for any $f \neq 0$. That $K f$ is positive for positive $f$, follows from the general theory outlined above, and also from:

$$
\langle\psi, K(f) \psi\rangle=2 \int_{\pi} f(x)\left|\psi^{\prime}(x)\right|^{2} d x .
$$

\section{Examples}

Example 6.1. If $G=\mathbb{T}, \mathscr{A}$ is the CAR algebra and $\tau$ is the gauge action, then if $\omega$ is a Powers' state, $\pi_{\omega}\left(\mathscr{A}^{\tau}\right)^{\prime \prime}$ is a factor and corollary 5.8 applies.

Example 6.2. If $G=\mathbb{T}, \mathscr{A}=\mathscr{O}_{n}$, and $\tau$ is the gauge action, then $\mathscr{A}^{\tau}$ is UHF, hence simple [12], and so corollary 5.8 applies.

Example 6.3. If $G=\mathbb{T}^{n}, \mathscr{A}=\mathscr{O}_{n}$ and $\tau$ is the gauge action, then $\mathscr{A}^{\tau}$ is isomorphic to the fixed point algebra of $\otimes M_{n}$ under the canonical action of $\mathbb{T}^{n-1}$ (c.f. example 6.1 for $n=2$ ). Then by the natural extension of 'Powers' states' (indexed by the interior of the $n$-simplex instead of $(0,1))$, from $\otimes M_{n}$ to $\mathscr{O}_{n}$, we can easily find a state $\omega$ of $\mathscr{O}_{n}$ such that $\pi_{\omega}\left(\mathscr{A}^{\top}\right)^{\prime \prime}$ is a factor. Thus corollary 5.8 applies.

Example 6.4. We analyze in detail ergodic actions of $G=\mathbb{Z}_{2} \times \mathbb{Z}_{2}$ on a simple $C^{*}$-algebra $\mathscr{A}$. This example will show that one-parameter semigroups of positive maps, commuting with an ergodic compact abelian action, are not necessarily completely positive.

Up to isomorphism, there is only one action of the stated type: $\mathscr{A}=M_{2}$, and if $g_{1}, g_{2}$ are the canonical generators of $G$, define

$$
\tau\left(g_{1}\right)=\operatorname{Ad}\left[\begin{array}{rr}
1 & 0 \\
0 & -1
\end{array}\right], \quad \tau\left(g_{2}\right)=\operatorname{Ad}\left[\begin{array}{ll}
0 & 1 \\
1 & 0
\end{array}\right] .
$$

The dual group $\hat{G}$ is isomorphic to $G$, and generated by the two characters $\gamma_{1}, \gamma_{2}$, given by

$$
\left\langle\gamma_{i}, g_{j}\right\rangle=(-1)^{i-j}
$$

Put $\gamma_{0}=0, \gamma_{3}=\gamma_{1}+\gamma_{2}$. The spectral subspaces $\mathscr{A}^{\tau}(\gamma)$ are one dimensional, and spanned by the unitaries $U(\gamma)$ given by

$$
\begin{array}{ll}
U\left(\gamma_{0}\right)=\left[\begin{array}{ll}
1 & 0 \\
0 & 1
\end{array}\right], & U\left(\gamma_{1}\right)=\left[\begin{array}{rr}
1 & 0 \\
0 & -1
\end{array}\right], \\
U\left(\gamma_{2}\right)=\left[\begin{array}{ll}
0 & 1 \\
1 & 0
\end{array}\right], & U\left(\gamma_{3}\right)=\left[\begin{array}{rr}
0 & 1 \\
-1 & 0
\end{array}\right] .
\end{array}
$$


If $\exp (-t H)$ is a semigroup commuting with $\tau$, the generator $H$ must map the spectral subspaces into themselves, i.e., there exist constants $\lambda_{n}$ such that

$$
H U\left(\gamma_{n}\right)=\lambda_{n} U\left(\gamma_{n}\right), \quad \text { for } n=0,1,2,3 .
$$

The action of $\exp (-t H)$ is then given by

$$
\begin{aligned}
& \exp (-t H)\left[\begin{array}{ll}
a & b \\
c & d
\end{array}\right] \\
= & \left\{(a+d)\left[\begin{array}{cc}
\exp \left(-t \lambda_{0}\right) & 0 \\
0 & \exp \left(-t \lambda_{0}\right)
\end{array}\right]+(a-d)\left[\begin{array}{cc}
\exp \left(-\lambda_{1} t\right) & 0 \\
0 & -\exp \left(-\lambda_{1} t\right)
\end{array}\right]\right. \\
& \left.+(b+c)\left[\begin{array}{cc}
0 & \exp \left(-\lambda_{2} t\right) \\
\exp \left(-\lambda_{2} t\right) & 0
\end{array}\right]+(b-c)\left[\begin{array}{cc}
0 & \exp \left(-\lambda_{3} t\right) \\
-\exp \left(-\lambda_{3} t\right) & 0
\end{array}\right]\right\} / 2 .
\end{aligned}
$$

To check when $\exp (-t H)$ is positivity preserving, it is enough to check when $\exp (-t H) E \geq 0$ for one-dimensional projections $E$, i.e. it is enough to verify that

$$
\operatorname{Tr}\left(\exp (-t H)\left[\begin{array}{cc}
1 & \gamma \\
\bar{\gamma} & |\gamma|^{2}
\end{array}\right]\right) \geq 0, \quad \operatorname{Det}\left(\exp (-t H)\left[\begin{array}{cc}
1 & \gamma \\
\bar{\gamma} & |\gamma|^{2}
\end{array}\right]\right) \geq 0
$$

for all $\gamma \in \mathbb{C}, t>0$. This gives the result that $\exp (-t H)$ is positivity preserving if and only if $\lambda_{i} \in \mathbb{R}$, for $i=0,1,2,3$ and

$$
\lambda_{i} \geq \lambda_{0}, \quad i=1,2,3 .
$$

Next we determine when $\exp (-t H)$ is completely positive. By [19], [24] this is the case if and only if $H$ has the form

$$
-H(A)=K(A)-L A-A L^{*}, \quad A \in M_{2},
$$

where $K$ is completely positive, $L \in M_{2}$. If $H$ is $\tau$-invariant, we may, by applying a mean, assume that $K$ and $L$ are $\tau$-invariant, and so $L$ is a scalar. If we normalize $H$ so that $H(1)=0$, we have

$$
-H(A)=K(A)-K(1) A, \quad \text { where } K(1) \in \mathbb{R} 1 .
$$

As $K$ is completely positive, it has the form

$$
K(A)=\sum_{i, j=0}^{3} \alpha_{i j} \sigma_{i} A \sigma_{j}
$$

where $\sigma_{0}=1, \sigma_{1}, \sigma_{2}, \sigma_{3}$ are the Dirac matrices, and $\left[\alpha_{i j}\right]$ is a positive matrix. As $K$ commutes with $G$, it actually has the form

i.e.

$$
K(A)=\sum \alpha_{i} \sigma_{i} A \sigma_{i}
$$

$$
-H(A)=\sum_{i=1}^{3} \alpha_{i} \sigma_{i} A \sigma_{i}-\left(\sum_{i=1}^{3} \alpha_{i}\right) A .
$$

Now $H\left(\sigma_{i}\right)=\lambda_{i} \sigma_{i}$ for $i=1,2,3$. This leads to the relations

$$
2 \alpha_{j}=\sum_{i \neq j} \lambda_{i}-\lambda_{j} \quad \text { for } j=1,2,3,
$$

and the summation index $i$ runs through $1,2,3$. As $\exp (-t H)$ is completely positive if and only if $\alpha_{j} \geq 0$ for $j=1,2,3$, this leads to the result that $\exp (-t H)$ is a 
completely positive semigroup if and only if

$$
\sum_{i \neq j} \lambda_{i} \geq \lambda_{j}, \quad \text { for } j=1,2,3 .
$$

(Here $\lambda_{0}=0$ as $H(1)=0$ ). Thus the set of completely positive semigroups commuting with $G$ is a proper subset of the positive semigroups commuting with $G$ in this case. See $[19]$ for a related result.

\section{Final remarks}

We expect that results similar to those in this paper should be true also for non-abelian compact groups $G$, but in this setting the requirement

$$
S \tau(g)=\tau(g) S
$$

is too strong. It has been suggested to us by $\mathrm{M}$. Takesaki that the two conditions

$$
S \tau(g)=\tau(g) S \text { and }\left.S\right|_{\mathscr{A}^{\tau}}=\text { id }
$$

could possibly be replaced by the single condition that each $G$-invariant closed subspace of $\mathscr{A}$ is $S$-invariant, see [1, appendix C]. However, the results do not extend to non-compact $G$ because the action of such groups does not in general have the correct smoothness properties. One simple example is $G=\mathbb{R}$ acting as an ergodic Kronecker flow on $C\left(\mathbb{T}^{2}\right)$, where $\mathbb{T}^{2}$ is the 2-torus. Then any element in $\mathbb{T}^{2}$ defines a completely positive map $S$ by transposition of the corresponding left translation on $\mathbb{T}^{2}$, and this map $S$ satisfies the requirements (4.1) in theorem 4.2. However, $S$ will only satisfy (4.2) if the element in $\mathbb{T}^{2}$ lies in the $\mathbb{B}$-orbit through 0 . Thus non-compact $G$ would require more hypotheses on $S$.

We are indebted to George A. Elliott for several enlightening remarks.

O. Bratteli was a Science and Engineering Research Council Senior Visiting Fellow during this research.

\section{REFERENCES}

[1] L. Accardi \& C. Cecchini. Conditional expectations in von Neumann algebras and a theorem of Takesaki. J. Func. Anal. 45 (1982), 245-273.

[2] H. Araki, R. Haag, D. Kastler \& M. Takesaki. Extensions of KMS states and chemical potential. Commun. math. Phys. 53 (1977), 97-134.

[3] W. B. Arveson. Subalgebras of $C^{*}$-algebras. Acta Math. 123 (1969), 141-224.

[4] S. Baaj. Multiplicateurs non Bornés. Paris preprint (1981).

[5] C. Berg \& G. Forst. Potential Theory on Locally Compact Abelian Groups. Springer-Verlag: Berlin-Heidelberg-New York, 1975.

[6] O. Bratteli \& P. E. T. Jørgensen. Unbounded derivations tangential to compact groups of automorphisms. J. Func. Anal. 48 (1982), 107-133.

[7] O. Bratteli \& D. W. Robinson. Operator Algebras and Quantum Statistical Mechanics I. SpringerVerlag: New York-Heidelberg-Berlin, 1979.

[8] O. Bratteli \& D. W. Robinson. Positive $C_{0}$-semigroups on $C^{*}$-algebras. Math. Scand. 49 (1981), 259-274.

[9] O. Bratteli, G. A. Elliott \& P. E. T. Jørgensen. Decomposition of unbounded derivations into invariant and approximately inner parts. Warwick preprint, (1982).

[10] L. G. Brown. Stable isomorphism of hereditary subalgebras of $C^{*}$-algebras. Pacific J. Math. 71 (1977), 335-348. 
[11] E. Christensen \& D. E. Evans. Cohomology of operator algebras and quantum dynamical semigroups. J. London Math. Soc. (2) 20 (1978), 358-368.

[12] J. Cuntz. Simple $C^{*}$-algebras generated by isometries. Commun. math. Phys. 57 (1977), 173-185.

[13] E. B. Davies. Some contraction semigroups in quantum probability. $Z$. Wahrscheinlichkeitstheorie verw. Geb. 23 (1972), 261-273.

[14] D. E. Evans. Irreducible quantum dynamical semigroups. Commun. math. Phys. 54 (1977), 293-297.

[15] D. E. Evans. Completely positive quasi-free maps on the CAR algebra. Commun. math. Phys. 70 (1979), 53-68.

[16] D. E. Evans \& J. T. Lewis. Dilations of irreversible evolutions in algebraic quantum theory. Comm. Dublin Inst. Adv. Studies, Ser. A 24 (1977).

[17] F. Goodman \& P. E. T. Jørgensen. Unbounded derivations commuting with compact group actions. Commun. math. Phys. 82 (1981), 399-405.

[18] F. Goodman \& A. Wasserman. Unbounded derivations commuting with compact group actions II. J. Funct. Anal. To appear.

[19] V. Gorini, A. Kossakowski \& E. C. G. Sudarshan. Completely positive dynamical semigroups on $N$-level systems. J. Math. Phys. 17 (1976), 821-825.

[20] A. Ikunishi. Derivations in $C^{*}$-algebras commuting with compact actions. Commun. math. Phys. To appear.

[21] P. E. T. Jørgensen. Compact symmetry groups and generators for sub-markovian semigroups. Z. Wahrscheinlichkeitstheorie verw. Greb. To appear.

[22] A. Kishimoto \& D. W. Robinson. On unbounded derivations commuting with a compact group of *-automorphisms. Commun. Math. Phys. To appear.

[23] A. Kishimoto \& H. Takai. Some remarks on $C^{*}$-dynamical systems with a compact abelian group. Publ. R.I.M.S. Kyoto Univ. 14 (1978), 383-397.

[24] G. Lindblad. On the generators of quantum dynamical semigroups. Commun. math. Phys. 48 (1976), 119-130.

[25] K. R. Parthasarathy \& K. Schmidt. Positive Definite Kernels, Continuous Tensor Products, and Central Limit Theorems of Probability Theory. Springer Lecture Notes No. 272, Springer-Verlag: Berlin-Heidelberg-New York, 1972.

[26] G. K. Pedersen. $C^{*}$-algebras and their automorphism groups. Academic Press: London-New York-San Francisco, 1979.

[27] C. Peligrad. Derivations of $C^{*}$-algebras which are invariant under an automorphism group I, OT series vol. 2, Birkhäuser Verlag (1981) II, Bucuresti preprint 107 (1981).

[28] R. T. Powers \& G. Price. Derivations commuting with $S(\infty)$. Commun. math. Phys. 84 (1982), $439-447$.

[29] D. W. Robinson. Strongly positive semigroups and faithful invariant states. Commun. math. Phys. 85 (1982), 129-142.

[30] I. E. Segal. A non-commutative version of abstract integration. Ann. Math. 57 (1953), $401-457$.

[31] M. H. Stone. On unbounded operators in Hilbert space. J. Indian. Math. Soc. 15 (1951), 155-192.

[32] M. Takesaki. Theory of Operator Algebras, I. Springer-Verlag: New York-Heidelberg-Berlin, 1979.

[33] S. Watanabe. Asymptotic behaviour and eigenvalues of dynamical semigroups on operator algebras. Niigata preprint. 